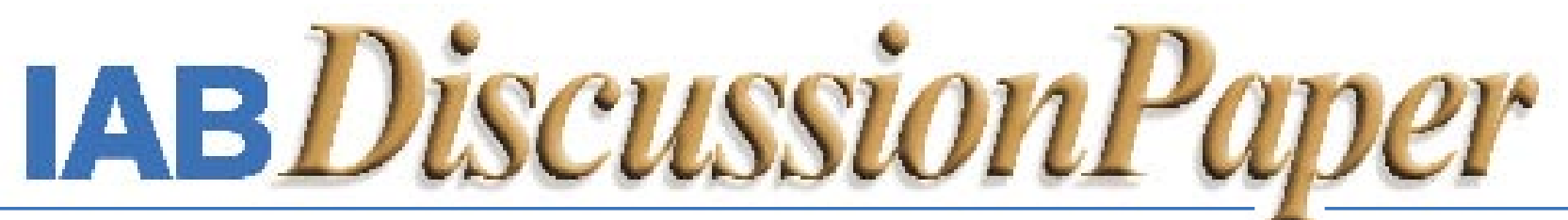

Beiträge zum wissenschaftlichen Dialog aus dem Institut für Arbeitsmarkt- und Berufsforschung

No. $24 / 2006$

\title{
Was spricht eigentlich gegen eine private Arbeitslosenversicherung?
}

Roman Lutz 


\title{
Was spricht eigentlich gegen eine private Arbeitslosenversicherung?
}

\author{
Roman Lutz
}

\begin{abstract}
Auch mit seiner neuen Reihe „IAB-Discussion Paper“ will das Forschungsinstitut der Bundesagentur für Arbeit den Dialog mit der externen Wissenschaft intensivieren. Durch die rasche Verbreitung von Forschungsergebnissen über das Internet soll noch vor Drucklegung Kritik angeregt und Qualität gesichert werden.
\end{abstract}

\begin{abstract}
Also with its new series "IAB Discussion Paper" the research institute of the German Federal Employment Agency wants to intensify dialogue with external science. By the rapid spreading of research results via Internet still before printing criticism shall be stimulated and quality shall be ensured.
\end{abstract}




\section{Inhaltsverzeichnis}

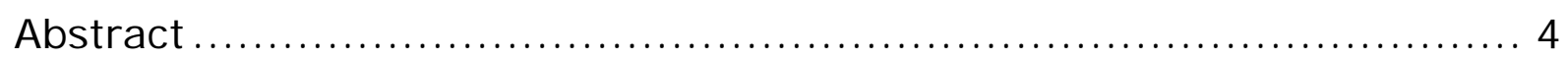

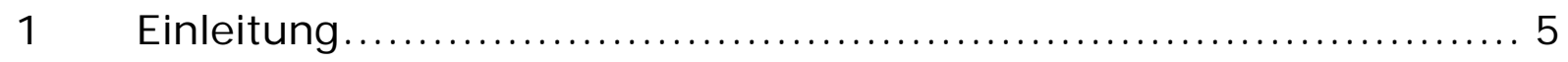

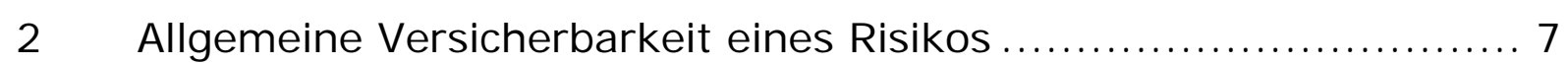

3 Versicherungstechnische Argumentation.......................... 14

3.1 Die individuellen Wahrscheinlichkeiten arbeitslos zu werden bzw. zu bleiben sind positiv korreliert .................................... 14

3.2 Probleme unvollständiger Information ............................ 26

3.2.1 Die individuellen Wahrscheinlichkeiten arbeitslos zu werden bzw. zu bleiben sind nicht hinreichend bekannt ..........................26

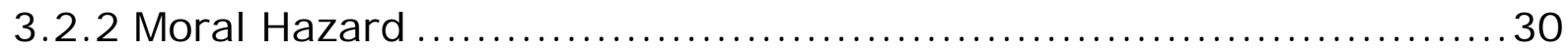

3.2.3 Versicherungsnehmer unterschätzen ihr individuelles Arbeitslosigkeitsrisiko ........................................... 39

$4 \quad$ Sozialpolitische Argumentation................................... 41

4.1 Die individuelle Wertschätzung einer Arbeitslosenversicherung ist bei Personen mit hohem Arbeitslosigkeitsrisiko zu gering

4.2 Für Personen mit hohem Arbeitslosigkeitsrisiko ist die finanzielle Belastung durch risikoäquivalente Prämien zu hoch.................42

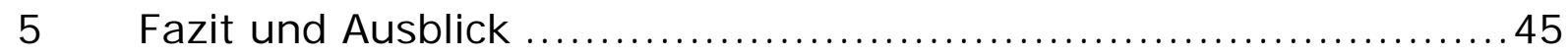

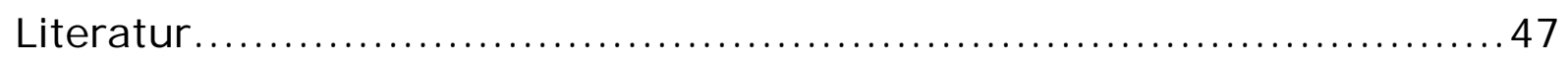




\section{Abstract}

Ziel der Arbeit ist die bisher fehlende versicherungsökonomische Fundierung in der Argumentation über die Privatisierung der Arbeitslosenversicherung. Anders als in bisherigen Ansätzen werden die Begründungen gegen eine Privatisierung in eine versicherungstechnische und eine sozialpolitische Perspektive zerlegt. Hierdurch soll die häufig zu beobachtende Vermengung von objektiv-technischen und normativen Argumenten aufgebrochen werden. Im Ergebnis wird herausgearbeitet, dass letztlich nur das normative Argument der "relativen Armut“ gegen eine private Arbeitslosenversicherung spricht. Demnach ist die Belastungswirkung risikoäquivalenter Prämien für Personen mit hohem bis mittlerem Arbeitslosigkeitsrisiko sozialpolitisch nicht vertretbar. Als wenig stichhaltig erweisen sich dagegen versicherungstechnische Argumente wie positive Korrelation der Einzelrisiken, Moral Hazard, unzureichende Prämiendifferenzierung sowie kollektive Unterschätzung des individuellen Arbeitslosigkeitsrisikos. Als Lösung für das Problem der "relativen Armut" wird ein neuer Entwurf zur Privatisierung der Arbeitslosenversicherung vorgestellt. Zentrales Element ist hierbei die kombinierte Versicherung von Arbeitslosigkeit und Langlebigkeit in einem Versicherungsprodukt.

JEL-Codes: G22, J65 


\section{Einleitung}

Lange Jahre galt das Risiko „Arbeitslosigkeit“ als nicht privat versicherbar und auch heute noch gibt es zahlreiche Vertreter dieser Meinung. ${ }^{1}$ Vergleichsweise einfach machen es sich dabei Autoren wie Rürup (2005: 377) sowie Berthold/von Berchem (2002: 67). Die Beobachtung, dass es bisher nirgendwo auf der Welt eine umfassende private Arbeitslosenversicherung gibt, erscheint innen als Indiz für die Unversicherbarkeit dieses Risikos. Zum einen jedoch entwickelt sich das Versicherungswesen ständig weiter, und zum anderen verdrängen die staatlichen Aktivitäten auf dem Feld der sozialen Sicherung ein privates Angebot. So lässt die umfangreiche gesetzliche Arbeitslosenversicherung in Deutschland kaum Freiräume für private Anbieter (Schäfer 2006: 26 sowie Schneider et al. 2004: 26). Das gegenwärtige Fehlen einer privaten Arbeitslosenversicherung kann also keineswegs als Argument gegen eine Privatisierung fungieren.

Tiefer greifendere Argumente in diesem Zusammenhang entspringen der Theorie des Marktversagens. Demnach sollen private Versicherungsmärkte nicht in der Lage sein, einen umfassenden Schutz bei Arbeitslosigkeit anzubieten. In jüngerer Zeit gibt es jedoch vermehrt Bemühungen, die Fachwelt vom Gegenteil zu überzeugen. ${ }^{2}$ Auch hierbei könnte man es sich einfach machen und lediglich die versicherungsökonomische Literatur zitieren. So erkennt beispielsweise Karten (1972: 287), „...daß alle Risiken prinzipiell versicherbar sind." Sehr eindringlich beschreiben auch Borch et al. (1990: 335) das Problem der Versicherbarkeit: „....actuaries present 20 papers which together included 400 pages laying down different conditions which a risk must meet in order to be insurable. All these sets of conditions make it impossible to insure against the capture of a monster in Loch Ness, but still the insurance was written." Natürlich sind diese pauschalen Einsichten in der Diskussion um die Ausgestaltung der Arbeitslosenversicherung wenig hilfreich, so dass man letztlich nicht umhin kommen wird, sich dezidiert mit den Problemen einer privaten Arbeitslosenversicherung auseinanderzusetzen.

1 Zu den prominentesten zählen Barr (1992 und 2001), Berthold (1988), Berthold/von Berchem (2002 und 2004), Berthold/Külp (1987), Eekhoff (1996), Rürup (2005), Sesselmeier et al. (2006).

2 Neben den Arbeiten von Glismann/Schrader (2005) sowie Beenstock/Brasse (1986) ist hier vor allem Schäfer (2006) zu nennen. 
Grundsätzlich ist festzustellen, dass es nur sehr wenige Befürworter einer privaten Arbeitslosenversicherung gibt, die sich auch die Mühe machen, ausführlich auf die zahlreichen Argumente gegen eine Privatisierung einzugehen. Beenstock/Brasse (1986) sowie Beenstock (1985) beispielsweise befassen sich überhaupt nicht damit und auch Beenstock (1986) beschränkt sich auf eine sehr rudimentäre Auseinandersetzung mit dem Thema. Glismann/Schrader (2005 und 2003) diskutieren zwar alle relevanten Argumente sehr ausführlich, und in komprimierter Form tut dies auch Schäfer (2006 sowie 2003a); jedoch bleiben sie meist eine an der Versicherungspraxis ausgerichtete Perspektive schuldig. Ganz allgemein fällt außerdem auf, dass sich die zahlreichen Analysen zur Privatisierung der Arbeitslosenversicherung - egal ob befürwortend oder ablehnend lediglich auf eine mehr oder weniger vollständige Aneinanderreihung der Einzelargumente beschränken, ohne auf deren theoretische Fundierung näher einzugehen. ${ }^{3}$ Implizit wird jedoch meist über die Versicherungstechnik argumentiert, wodurch der Eindruck entsteht, als wäre das Arbeitslosigkeitsrisiko gerade aufgrund versicherungstechnischer Unzulänglichkeiten nicht privat versicherbar. ${ }^{4}$

In den folgenden Abschnitten wird deshalb zunächst ein Rahmen aus allgemeiner versicherungstheoretischer Sicht gespannt, um die Auseinandersetzung mit den kursierenden Argumenten versicherungsökonomisch zu fundieren. Anders als in bisherigen Ansätzen werden anschließend die Begründungen gegen eine Privatisierung der Arbeitslosenversicherung in eine versicherungstechnische und eine sozialpolitische Perspektive zerlegt. Hierdurch soll die in der Literatur häufig zu beobachtende Vermengung von objektiv-technischen und normativen Argumenten aufgebrochen werden. Im Ergebnis soll herausgearbeitet werden, welche der beiden Argumentationen tatsächlich gegen eine private Arbeitslosenversicherung spricht. Um die Realisierbarkeit einer privaten Arbeitslosenversicherung aus objektiv-technischer Sicht zu erörtern, wird explizit auf risikopolitische

3 Z. B. Glismann/Schrader (2005: 59 ff.), Knappe (1995: 349 ff.) sowie Schäfer (2006: 31 f., 2003a: 43 ff. sowie 2003b: 232). Eine Ausnahme bildet die Arbeit von Schönbäck (1988), die die Versicherbarkeit des Arbeitslosigkeitsrisikos erwartungsnutzentheoretisch motiviert.

4 Beispielhaft sei hier auf Barr (2001: 35 ff.), Berthold/Külp (1987: $77 \mathrm{ff}$.), Berthold/von Berchem (2004: 4 ff. sowie 2002: 59 ff.), Bruttel (2005: 292), Eekhoff (1996: 154 f.), Sachverständigenrat (2003: 393), Sesselmeier et al. (2006: 13 ff.) verwiesen. 
Instrumente und Versicherungstechnik eingegangen. Damit wird eine weitere Lücke in der ökonomischen Literatur zur Privatisierung der Arbeitslosenversicherung geschlossen.

\section{Allgemeine Versicherbarkeit eines Risikos}

Die Frage nach der Versicherbarkeit von Risiken kann grundsätzlich von zwei Seiten angegangen werden. Die eine ist versicherungstechnisch und an der Beschaffenheit des zu versichernden Risikos orientiert. Die andere ist entscheidungstheoretisch fundiert und argumentiert über das Zustandekommen eines Versicherungsmarktes. Es zeigt sich jedoch, dass diese beiden Sichtweisen letztendlich aufeinander aufbauen und nicht getrennt betrachtet werden können. So ist die versicherungstechnische Perspektive auf das Versicherungsangebot fokussiert. Die Entscheidungstheorie bezieht neben dem Angebot auch die Nachfrage mit ein. Danach ist ein Risiko dann versicherbar, wenn sich Angebot und Nachfrage über den Preis für Versicherungsschutz ausgleichen. Ein potentieller Versicherungsnehmer wird dann Versicherungsschutz nachfragen, wenn die zu bezahlende Bruttoprämie (P) kleiner ist als die Summe aus Erwartungswert seiner individuellen Schadenverteilung $[E(S)]$ und subjektivem Risikopreis ( $r$ ). Hinter Letzterem verbirgt sich derjenige Betrag, den ein Versicherungsnehmer über den reinen Schadenerwartungswert hinaus für die Deckung seines Risikos bereit ist zu bezahlen. Ist dieser Betrag positiv, so wird dadurch eine Abneigung gegen Risiko impliziert. Insofern ist der subjektive Risikopreis ein Maß für die Risikoaversion des Versicherungsnehmers. ${ }^{5}$ Es ist davon auszugehen, dass bei hoher erwarteter Schadenvolatilität auch der subjektive Risikopreis vergleichsweise hoch ausfällt (Berliner 1982: 59). Insofern steht die Streuung der Schadenverteilung in positivem Zusammenhang mit dem subjektiven Risikopreis. Eine weitere Einflussgröße auf das Ausmaß der Risikoaversion ist der individuelle Nutzen einer Versicherung [U(V) ${ }^{\mathrm{s}}$ ] im Vergleich zum individuellen Nutzen aus alternativen Verwendungsarten $\left[U(A)^{s}\right] .{ }^{6}$ Der subjektive Risikopreis hängt

5 Die Differenz aus Erwartungswert der Schadenverteilung und subjektivem Risikopreis ergibt das Sicherheitsäquivalent. Dieses ist der derjenige sichere Betrag, der gerade denselben Nutzen stiftet wie die unsichere Risikosituation (Karten 1972: 282).

6 Zur Vereinfachung geht die Nutzendifferenz an dieser Stelle direkt in die Risikoaversion ein. Die Beziehung zwischen Nutzen aus Versicherung und alternativer Verwen- 
somit auch von den Präferenzen für andere Güter als der Versicherung ab und kann letztlich vereinfacht als Funktion der Schadenvarianz und Nutzendifferenz interpretiert werden.

Ein Versicherer wird dann Versicherungsschutz anbieten, wenn die Bruttoprämie ( $P$ ) einer Risikogruppe mindestens die Summe aus Schadenerwartungswert $[E(S)]$ und Kostenzuschlag $(k)$ dieser Risikogruppe deckt, wenn also sein Nutzen aus der Prämie größer ist als der Missnutzen aus der übernommenen Schadenverteilung (Karten 1972: 283). Neben anderen Einflussfaktoren $(z)^{7}$ ist der Kostenzuschlag eine Funktion der Schadenvarianz $[\operatorname{Var}(\mathrm{S})]$, welche sich bei der Prämienkalkulation im Sicherheitszuschlag wieder findet. Ein Risiko ist demnach dann versicherbar, wenn eine Prämie existiert, die folgenden Wertebereich aufweist: ${ }^{8}$

$$
\mathrm{E}(\mathrm{S})+\mathrm{k}[\operatorname{Var}(\mathrm{S}) ; \mathrm{Z}] \leq \mathrm{P} \leq \mathrm{E}(\mathrm{S})+r\left[\operatorname{Var}(\mathrm{S}) ; \mathrm{U}(\mathrm{V})^{\mathrm{s}}-\mathrm{U}(\mathrm{A})^{\mathrm{s}}\right]
$$

In den bisherigen Überlegungen wurde angenommen, dass Erwartungswert und Varianz der Schadenverteilung für Anbieter und Nachfrager identisch ist. Informationsasymmetrien und Heterogenität der Risikogruppen können jedoch zu abweichenden Vorstellungen bezüglich der Schadenverteilungen führen. So kann der Versicherungsnehmer (VN) von geringeren Werten ausgehen als das Versicherungsunternehmen (VU). Genauso gut kann natürlich auch der umgekehrte Fall eintreten, wenngleich dies unproblematisch in Bezug auf die Versicherbarkeit ist. Das bedeutet also, die Schadenverteilung setzt sich nicht aus objektiven Komponenten zusammen, sondern hängt von den jeweiligen subjektiven Einschätzungen (s) der beteiligten Akteure ab (Eisen 1988: 121). Der für die Versicherbarkeit eines Risikos erforderliche Wertebereich der Prämie ist nun genauer definiert als:

$$
\mathrm{E}(\mathrm{S})_{\mathrm{VU}}^{\mathrm{s}}+\mathrm{k}\left[\operatorname{Var}(\mathrm{S})_{\mathrm{VU}}^{\mathrm{s}}\right] \leq \mathrm{P} \leq \mathrm{E}(\mathrm{S})_{\mathrm{VN}}^{\mathrm{s}}+\mathrm{r}\left[\operatorname{Var}(\mathrm{S})_{\mathrm{VN}}^{\mathrm{s}} ; \mathrm{U}(\mathrm{V})^{\mathrm{s}}-\mathrm{U}(\mathrm{A})^{\mathrm{s}}\right]
$$

dungsart kann auch als eine eigenständige Einflussgröße auf die Zahlungsbereitschaft eines Versicherungsnachfragers modelliert werden.

7 Darunter werden Zuschläge für Verwaltungs- und Abschlusskosten, Gewinn, ggf. Steuern und ggf. eine Sparprämie subsumiert.

8 Kostenzuschlag (k) bzw. subjektiver Risikopreis ( $r$ ) sind jeweils als Funktion der Schadenvarianz [ $\operatorname{Var}(S)]$ sowie anderer Kostenfaktoren ( $z$ ) bzw. subjektiver Nutzendifferenz $\left[U(V)^{s}-U(A)^{s}\right]$ zu interpretieren. 
Es zeigt sich an dieser Stelle, dass Risikoaversion beim Versicherungsnehmer keine notwendige Bedingung für das Zustandekommen eines Versicherungskontraktes ist. ${ }^{9}$ Wenn der vom Versicherungsnehmer unterstellte Schadenerwartungswert größer ist als die Prämie, die der Versicherer mindestens verlangen muss, kann es auch bei risikoneutraler bzw. risikofreudiger Einstellung des Versicherungsnehmers zu einer Versicherung kommen. Dies steht scheinbar im Widerspruch zu den Ausführungen von Karten (1972: 282) oder Schönbäck (1988: 49), die die Risikoaversion als notwendig dafür ansehen, dass die Versicherungsnahme überhaupt erwogen wird. Allerdings wird in den genannten Arbeiten davon ausgegangen, dass Versicherungsnehmer und Versicherer vollständig informiert sind und somit von der gleichen (wahren) Schadenverteilung ausgehen.

Letztendlich muss noch die Budgetrestriktion berücksichtigt werden. Selbst wenn ein Versicherungsnehmer bereit ist, eine bestimmte Prämie zu entrichten, muss er dennoch die finanzielle Ausstattung ( $Y$ ) hierfür besitzen. Das heißt, die Prämie darf in keinem Fall einen Wert übersteigen, bei dessen Entrichtung der Versicherungsnehmer das Existenzminimum ( $Y_{\text {MIN }}$ ) unterschreitet (Schönbäck 1988: 49). Der zulässige Wertebereich der Prämie beträgt schließlich:

$$
\mathrm{E}(\mathrm{S})_{\mathrm{VU}}^{\mathrm{s}}+\mathrm{k}\left[\operatorname{Var}(\mathrm{S})_{\mathrm{VU}}^{\mathrm{s}}\right] \leq \mathrm{P} \leq \operatorname{Min}\left\langle\left(\mathrm{Y}-\mathrm{Y}_{\mathrm{MIN}}\right) ;\left\{\left(\mathrm{E}(\mathrm{S})_{\mathrm{VN}}^{\mathrm{s}}+\mathrm{r}\left[\operatorname{Var}(\mathrm{S})_{\mathrm{VN}}^{\mathrm{s}} ; \mathrm{U}(\mathrm{V})^{\mathrm{s}}-\mathrm{U}(\mathrm{A})^{\mathrm{s}}\right]\right\}\right\rangle\right.
$$

Hinter der Summe $E(S)_{v u}^{s}+k\left[\operatorname{Var}(S)_{v u}^{s}\right]$ verbirgt sich die hypothetische Schadenverteilung einer Risikogruppe, die das Versicherungsunternehmen zur Kalkulation der Prämie für diese Risikogruppe unterstellt. Zu beachten ist jedoch, dass der Versicherer nicht die hypothetische, sondern die tatsächliche Schadenverteilung in Deckung nimmt. Zusammenfassend können nun folgende Faktoren identifiziert werden, die die Versicherbarkeit eines Risikos bestimmen:

1) Die Präferenz für eine Versicherung im Vergleich zu alternativen Verwendungsarten muss stark genug sein, damit - über die Risikoaversion - die Zahlungsbereitschaft der potentiellen Versicherungsnehmer mindestens der Prämie entspricht.

9 Wenngleich im Allgemeinen von risikoaversen Akteuren ausgegangen werden kann (Eisen/Zweifel 2003: 43). 
2) Die finanzielle Ausstattung der Versicherungsnehmer muss ausreichen, um nach Abzug der Versicherungsprämie das Existenzminimum nicht zu unterschreiten.

3) Die subjektiven Einschätzungen der Versicherungsnehmer bezüglich ihrer individuellen Schadenverteilungen dürfen nicht zu optimistisch sein im Vergleich zur hypothetischen Schadenverteilung der Risikogruppe, die der Versicherer unterstellt.

4) Die Abweichung zwischen tatsächlicher und hypothetischer Schadenverteilung des gesamten Versichertenkollektivs darf ein für den Versicherer tragbares Ausmaß nicht übersteigen.

Die erste Bedingung ist an sich nicht problematisch. Sie führt lediglich da$z u$, dass nicht alle Personen einen Versicherungsschutz nachfragen. Nur eine zu geringe relative Wertschätzung der Versicherung bei einem Großteil der potentiellen Versicherungsnehmer lässt einen Versicherungsmarkt scheitern. Für das Risiko „Arbeitslosigkeit“ ist allerdings nicht davon auszugehen. Ansonsten hätte es kaum einen gesellschaftlichen Konsens gegeben, der zur Einführung einer gesetzlichen Arbeitslosenversicherung geführt hat. ${ }^{10}$ Es könnte jedoch systematische Unterschiede in der Bewertung einer Arbeitslosenversicherung geben, wenn die diesbezügliche Wertschätzung negativ korreliert ist mit der risikoäquivalenten Prämie. Dann würden Personen mit hohem Arbeitslosigkeitsrisiko keine Versicherung nachfragen. Diese Situation stellt kein Problem für das Versicherungsgewerbe dar, muss aber im Hinblick auf gesellschaftliche Vorstellungen diskutiert werden. Dies geschieht in Abschnitt 4.1.

Geht man davon aus, dass das Existenzminimum eine vorgegebene Größe ist, so bezieht sich die zweite Bedingung auf das Budget eines Versicherungsnehmers in Bezug auf seine risikoäquivalente Prämie. Aus versicherungstechnischer Sicht ist auch hier lediglich der Extremfall problematisch, dass sich niemand eine Versicherung leisten kann, also überhaupt keine Nachfrage existiert. Dies ist jedoch bei einer Versicherung von Arbeitslosigkeit nicht zu befürchten. Allerdings ist eine negative Korrelation zwi-

10 Die Untersuchung von Ulrich (2006) hat gezeigt, dass die Mehrheit der Deutschen ein höheres Arbeitslosengeld befürwortet. Dies kann als Indiz dafür erachtet werden, dass eine Versicherung des Arbeitslosigkeitsrisikos hohe individuelle Wertschätzung in der Gesellschaft erfährt. 
schen Budget und risikoäquivalenter Prämie empirisch zu beobachten (Biewen/Wilke 2005: 10 sowie 30 f.). Somit besteht die Möglichkeit, dass Personen mit hohem Arbeitslosigkeitsrisiko keine Versicherung finanzieren können. Dies ist jedoch kein versicherungstechnisches Problem, sondern eine Frage der Bewertung. Deshalb wird dieser Zusammenhang in Abschnitt 4.2 thematisiert.

Die dritte Bedingung ist unter den Aspekt der Informationsproblematik einzuordnen. Sofern die Versicherungsnehmer ihr individuelles Risiko systematisch unterschätzen, kann es zu einer Unversicherbarkeit kommen. Wie bei den beiden vorangegangenen Bedingungen ist dies nur dann der Fall, wenn ein Großteil des potentiellen Versichertenkollektivs betroffen ist, wenn das Risiko also kollektiv unterschätzt wird. Im Zusammenhang mit dem Arbeitslosigkeitsrisiko wird dies in der Literatur immer wieder angeführt, weshalb in Abschnitt 3.2.3 näher darauf eingegangen wird. Außerdem kann die Heterogenität der Risikogruppe dazu führen, dass der einzelne Versicherungsnehmer sein Risiko anders einschätzt als der Versicherer. Die Ursache hierfür liegt allerdings nicht beim Versicherungsnehmer, sondern ist in der unzureichenden Prämiendifferenzierung des Versicherers zu finden. Dieser Zusammenhang wird in Abschnitt 3.2.1 unter dem Stichwort "adverse Selektion“ thematisiert.

Der vierte Punkt bezieht sich auf die Gesamtschadenverteilung des Versichertenkollektivs und nicht auf Schadenverteilungen einzelner Risikogruppen, da eine Quersubventionierung zwischen unterschiedlichen Risikogruppen für den Versicherer stets möglich ist. An dieser Stelle geht es um das langfristige Überleben eines Versicherungsunternehmens. Dies ist nur dann gewährleistet, wenn die gesamten Bruttobeitragseinnahmen die Bruttoausgaben inklusive Schadenaufwendungen decken. Von zentralem Interesse ist hierbei das versicherungstechnische Risiko, welches letztlich durch die Beschaffenheit der zu versichernden Einzelrisiken bestimmt wird. Das versicherungstechnische Risiko ist dasjenige Risiko, welches vom Versicherer zu tragen ist und über das reine Unternehmerrisiko hinausgeht. Es bezeichnet die Ungewissheit über den wahren Schadenaufwand des Versichertenkollektivs in der Zukunft (Helten/Karten 
1984: 183). ${ }^{11}$ Wie eingangs bereits erwähnt, wird nun deutlich, dass das Zustandekommen eines Versicherungsmarktes über den Ausgleich von Versicherungsangebot und - nachfrage auch direkt von den Eigenschaften des Risikos abhängt. In diesem Zusammenhang nennt Karten (1972: 287) fünf Anforderungen, die an ein zu versicherndes Risiko gestellt werden: ${ }^{12}$

- Größe

- Zufälligkeit

- Eindeutigkeit

- Schätzbarkeit

- Unabhängigkeit

Das Kriterium der Größe fordert, dass die maximal fällige Versicherungsleistung für ein Einzelrisiko nicht aus dem übrigen Kollektiv herausragen sollte, da hierdurch der Risikoausgleich gestört wird (Karten 1972: 292). Durch Begrenzung der Schadensummen ist es jedoch möglich, ein bestehendes Risikogrößenproblem zu beseitigen. Auch bei der Versicherung von Arbeitslosigkeit ist dieses Kriterium somit nicht von Bedeutung.

Zufälligkeit verlangt, dass der Versicherungsfall ungewiss und unbeeinflussbar ist. Hier wird offensichtlich die Moral Hazard Problematik angesprochen, welche im Zusammenhang mit einer privaten Arbeitslosenversicherung starke Aufmerksamkeit erfährt und deshalb in Abschnitt 3.2.2 ausführlich behandelt wird.

Eindeutigkeit sollte in der Relation Schadenfall - Versicherungsleistung bestehen. Es sollte also eindeutig sein, welche Leistung bei welchem Schadenereignis vom Versicherer zu erbringen ist. Diese Forderung ist im Allgemeinen unproblematisch, da der Versicherungsvertrag dahingehend gestaltbar ist. Insofern ist der Hinweis von Sesselmeier et al. (2006: 26), dass die tatsächliche Schadenhöhe von Arbeitslosigkeit nicht monetär be-

${ }^{11}$ Zu der Unterteilung des versicherungstechnischen Risikos in Diagnose- und Prognoserisiko sei auf Helten/Karten (1984: $186 \mathrm{ff}$.) verwiesen. Farny (2006: $83 \mathrm{ff}$.) unterscheidet zwischen Zufallsrisiko, Änderungsrisiko und Irrtumsrisiko.

12 Es wird hier auf den Kriterienkatalog von Karten (1972) eingegangen, da dieser in der Literatur sehr etabliert ist. Eine weitere oft zitierte Unterscheidung geht auf Berliner (1982) zurück. Sehr detailliert ist die Untergliederung in Swiss Re (2005: 7 f.). Im Kern sprechen die unterschiedlichen Kataloge dieselben Kriterien für Versicherbarkeit an. Sie unterscheiden sich lediglich in der Feinheit der Untergliederung. 
wertbar ist, für die private Versicherbarkeit des Arbeitslosigkeitsrisikos belanglos (Schönbäck 1988: 49).

Schätzbarkeit verlangt, dass der Versicherer die Gesamtschadenverteilung eines Kollektivs hinreichend genau erfassen kann, um daraus den Schadenbedarf in der Zukunft ableiten zu können. Die Abweichungen zwischen Prognose und wahrem Wert sollten so gering wie möglich ausfallen. Sofern aus der Varianz der hypothetischen Gesamtschadenverteilung bereits eine starke Prognoseabweichung absehbar ist (Zufallsrisiko), kann der Versicherer durch hohe Sicherheitszuschläge dennoch vergleichsweise solide kalkulieren. Problematischer sind Änderungen des Risikoursachensystems (Änderungsrisiko). Diese sind in ihrer Wirkung nicht vorhersehbar und somit für den Versicherer nicht kalkulierbar. Karten (1972: 290) sowie Helten/Karten (1984: 211) machen jedoch deutlich, dass die Versicherungspraxis die Wichtigkeit des Schätzbarkeitskriteriums bereits eingeschränkt hat. „Es werden immer wieder neue Risiken versichert, über die noch gar keine statistische Schadenerfahrung vorliegt. Der versicherungstechnische Risikoausgleich wird dadurch nicht beeinträchtigt" (Helten/ Karten 1984: 211). Dennoch wird bei der Argumentation zur Privatisierung der Arbeitslosenversicherung der Schätzbarkeit große Beachtung beigemessen, weshalb in Abschnitt 3.2.1 darauf eingegangen wird.

Das Kriterium der Schätzbarkeit ist eng mit dem der Unabhängigkeit verbunden. So lassen sich valide Prognosen nur dann berechnen, wenn die Einzelrisiken stochastisch unabhängig voneinander sind. In der Frage der Versicherbarkeit eines Risikos wird diese Forderung als die bedeutendste eingestuft (Karten 1972: 291), da bei abhängigen Risiken der Ausgleich im Kollektiv maßgeblich beeinflusst wird (Helten/Karten 1984: 212). Ob stochastisch abhängige Risiken versicherbar sind, hängt letztendlich vom Ausmaß der Versicherungsleistungen $a b$, das durch die Abhängigkeit der Einzelrisiken vom Versicherer geleistet werden muss (Karten 1972: 292) sowie von der Wirksamkeit der zur Verfügung stehenden risikopolitischen Instrumente. Im nächsten Abschnitt (3.1) wird dies im Zusammenhang mit der Arbeitslosenversicherung ausführlich erörtert. 


\section{Versicherungstechnische Argumentation}

\subsection{Die individuellen Wahrscheinlichkeiten arbeitslos zu werden bzw. zu bleiben sind positiv korreliert}

Für die private Versicherbarkeit des Arbeitslosigkeitsrisikos ist es unerlässlich, dass die Versicherungsunternehmen effektive Risikotransformation betreiben können. Dadurch wird erreicht, dass das kollektive Risiko einer Versichertengemeinschaft (Risikogruppe) geringer ist als die Summe der Einzelrisiken. Aus den Schadendaten einer ganzen Risikogruppe lassen sich bessere Prognosen berechnen, als aus den Angaben einzelner Versicherter. Der Grund dafür ist, dass sich der durchschnittliche Schaden einer Risikogruppe mit wachsender Gruppengröße einem festen Wert annähert (Swiss Re 2002: 6). Dieser Zusammenhang kann aus dem „Gesetz der großen Zahlen“ abgeleitet werden und wird auch durch das so genannte Wurzel-n-Gesetz beschrieben (Schlittgen 2003: 262): ${ }^{13}$

Sei $S_{i}^{A}$ eine identisch verteilte Zufallsvariable, die den zufällig eintretenden Schaden aus Arbeitslosigkeit des i-ten Versicherungsnehmers umschreibt. Dann ist der Gesamtschaden des Versichertenkollektives (der Risikogruppe) $\mathrm{S}=\sum_{1}^{\mathrm{n}} \mathrm{S}_{\mathrm{i}}^{\mathrm{A}}$ der Schaden, den die Versicherung tragen muss. Der Erwartungswert des Gesamtschadens aus Arbeitslosigkeit ist definiert als $E(S)=\sum_{1}^{n} E\left(S_{i}^{A}\right)=n \cdot E\left(S_{i}^{A}\right)$. Die Standardabweichung des Gesamtschadens beträgt $\sqrt{\operatorname{Var}(S)}=\sqrt{\operatorname{Var}\left(\sum_{1}^{n} S_{i}^{A}\right)}$ und stellt quasi das Risiko dar, dem sich das Versicherungsunternehmen ausgesetzt sieht. Nur für stochastisch unabhängige Einzelrisiken $S_{i}^{A}$ gilt, dass $\sqrt{\operatorname{Var}\left(\sum_{1}^{n} S_{i}^{A}\right)}=$ $\sqrt{\sum_{1}^{n} \operatorname{Var}\left(S_{i}^{A}\right)}=\sqrt{n \cdot \operatorname{Var}\left(S_{i}^{A}\right)}$. Dieses Gesamtrisiko steigt mit zunehmender Versichertenzahl. Das durchschnittlich auf einen Versicherungsnehmer anfallende Risiko beträgt jedoch $\frac{\sqrt{\operatorname{Var}\left(S_{i}^{A}\right)}}{\sqrt{n}}$ und sinkt mit steigender Zahl an Versicherungsnehmern. Somit wächst das Gesamtrisiko weniger als die Summe der Einzelrisiken (Meyer 1997: 18), was auch als versicherungs-

13 Helten/Karten (1984: 51) sprechen in diesem Zusammenhang vom Produktionsgesetz der Versicherungswirtschaft. 
technische economies of scale bezeichnet werden kann. Sinn (1986: 563) sieht darin eine Eliminierung von Risiko.

Vorraussetzung hierfür ist jedoch die stochastische Unabhängigkeit und damit Unkorreliertheit der Einzelrisiken. Die einzelnen Versicherungsnehmer müssen also unabhängig voneinander von Arbeitslosigkeit betroffen sein. Dies kann in der Realität jedoch zumindest nicht zu hundert Prozent beobachtet werden. Die individuellen Risiken arbeitslos zu werden bzw. zu bleiben sind positiv miteinander korreliert. Das bedeutet, Arbeitslosigkeit tritt gehäuft auf. Über die Verflechtungen innerhalb und zwischen Unternehmen sowie zwischen Branchen können zum einen konjunkturelle Schwankungen als Ursache für die Verbundenheit der Arbeitslosigkeitsrisiken identifiziert werden, zum anderen zeichnen sich strukturelle Veränderungen dafür verantwortlich (Barr 2001: 36 sowie Eekhoff 1996: 221). In der Versicherungsökonomie spricht man in diesem Zusammenhang von Kumul- und Ansteckungsrisiken sowie vom Risiko schwankender Grundwahrscheinlichkeiten (Helten/Karten 1984: 212). Für eine Arbeitslosenversicherung ist der Extremfall eines Kumuls in großen Wirtschaftskrisen zu sehen (Schönbäck 1988: 55). Die positive Korrelation der Arbeitslosigkeitsrisiken kann sich unterschiedlich auf eine Versicherung auswirken: Wirtschaftliche Entwicklungen können einerseits dazu führen, dass innerhalb kurzer Zeit viele Personen arbeitslos werden. Dadurch steigt die Schadenzahl und somit die Schadensumme des Versichertenkollektivs. Zweitens kann es durch strukturelle Veränderungen zu längeren Arbeitslosigkeitsdauern kommen. Hierdurch steigt zwar nicht die Schadenzahl, wohl aber die durchschnittliche Schadenhöhe, wodurch ebenfalls die Summe der versicherten Schäden steigt. Beide Szenarien können auch gleichzeitig eintreten. Dann steigt sowohl die Schadenszahl als auch die durchschnittliche Schadenhöhe. Ansteckung ${ }^{14}$ in Bezug auf das Arbeitslosigkeitsrisiko kann sowohl intrapersonell (Bender et al. 2000 sowie Arrow 1996) $)^{15}$, als auch interpersonell beobachtet werden. ${ }^{16}$ Letzteres hat seinen

${ }^{14}$ Glismann/Schrader (2005: 67) sprechen in diesem Zusammenhang von Infektionsrisiko.

${ }^{15}$ Dies ist jedoch unproblematisch im Hinblick auf die Versicherbarkeit des Arbeitslosigkeitsrisikos.

16 Der Unterscheidung von Sesselmeier et al. (2006: 23 ff.) in inter- und intratemporale sowie inter- und intrapersonelle Abhängigkeit wird hier nicht gefolgt, da sie wenig trennscharf ist. 
Ursprung z. B. in prozyklischem Verhalten der Tarifparteien (Schäfer 2003a: 44 sowie Glismann/Schrader 2005: 67).

Dem Versicherungsgewerbe stehen heute wirkungsvolle Instrumente zur Verfügung, um das Problem der stochastisch abhängigen Arbeitslosigkeitsrisiken in den Griff zu bekommen. Zunächst sei jedoch erwähnt, dass abhängige Risiken nicht per se unversicherbar sind. Je größer die Korrelation zwischen den versicherten Einzelrisiken, desto schwächer wirkt das oben beschriebene Gesetz der großen Zahlen. Somit sinkt das durchschnittliche Risiko pro Versicherungsnehmer mit zunehmender Versichertenzahl weniger stark (Berliner 1982: 50). Das Risiko, dem sich das Versicherungsunternehmen ausgesetzt sieht, ist bei positiv korrelierten Risiken ceteris paribus größer als bei unkorrelierten (oder negativ korrelierten) Risiken. Die Versicherbarkeit eines Risikos ist allerdings nur dann eingeschränkt, wenn die Korrelation zwischen den Risiken zu stark ausfällt bzw. gewisse Grenzen der Unabhängigkeit überschritten werden (Swiss Re 2005: 7; Mugler 1980: 77, ähnlich Barr 1992: 753). Ob die Grenzen der Unabhängigkeit in Bezug auf das Arbeitslosigkeitsrisiko überschritten sind, kann keineswegs eindeutig festgestellt werden.

Topel/Welch (1980: 356) bemessen die Korrelation des Arbeitslosigkeitsrisikos für ausgewählte amerikanische Staaten auf 50\%-60\% und schließen daraus, dass das Arbeitslosigkeitsrisiko nicht privat versicherbar, weil zu wenig diversifizierbar, ist. Aus diesen Ergebnissen leiten zahlreiche Arbeiten ${ }^{17}$ auch für Deutschland eine maximale Diversifizierbarkeit des Arbeitslosigkeitsrisikos von etwa $50 \%$ ab und gelangen zu demselben Schluss einer Nichtversicherbarkeit. Diese Schlussfolgerung ist jedoch insofern problematisch, als bei Topel/Welch (1980) nicht die wahre Korrelation zwischen den individuellen Arbeitslosigkeitsrisiken gemessen wird, sondern die durchschnittliche Korrelationen zwischen branchenbezogenen Arbeitslosigkeitsrisiken. Diversifizierungspotential innerhalb von Branchen wird damit nicht erfasst. ${ }^{18}$ Außerdem kann keineswegs gesagt werden, ob eine

17 Zu ihnen zählen unter anderem Berthold/Külp (1987: 78), Hartmann (1998: 179), Berthold/von Berchem (2002: 50) sowie von Berchem (2005: 87).

18 Topel/Welch (1980: 375 f.) weisen selbst darauf hin, dass die Aggregation in Branchen die wahre Korrelation überschätzt. 
Korrelation von 50\% zu hoch ist, um ein Risiko ausreichend zu diversifizieren. ${ }^{19}$

Beenstock (1986: 11) sowie Wagner/Jahn (2004: 228) sehen eine private Versicherung von Arbeitslosigkeit nur dann als unmöglich an, wenn die Risiken perfekt positiv korreliert sind. ${ }^{20}$ Dieser Fall tritt am ehesten im Zuge großer Wirtschaftskrisen ein. Hinzu kommt, dass derartige Ereignisse meist gänzlich unvorhersehbar sind. Glismann/Schrader (2005: 68) sehen in großen Wirtschaftskrisen dennoch kein Problem für die Versicherbarkeit des Arbeitslosigkeitsrisikos und verweisen hierzu auf Versicherungstechniken, mithilfe derer auch extrem hohe Risiken versichert werden können. Da die beiden Autoren kaum auf die einzelnen „Techniken“ eingehen, wird sich der nächste Absatz damit genauer befassen. Weiter führen Glismann und Schrader aus, dass auch eine staatliche Versicherung bei großen Wirtschaftskrisen überfordert wäre. Hierzu ist anzumerken, dass der Staat in Krisenzeiten auf Steuermittel und die Möglichkeit der Verschuldung zurückgreifen kann und somit größere Ressourcen zur Verfügung hat als private Versicherer (Nell/Richter 2003: 334). Schließlich verweisen sie darauf, dass nicht alle Risiken zu 100 Prozent abgesichert werden müssen und sich Wirtschaftskrisen somit nicht in vollem Umfang auf die Versicherung auswirken. Dieses Argument ist allerdings wenig stichhaltig. Franchisen $^{21}$ können nur dann zur Milderung von Kumulschäden sinnvoll eingesetzt werden, wenn es sich um Massenschäden mit geringem Einzelschaden handelt (Hartmann 1998: 133). Liegen viele Einzelschäden unterhalb der Selbstbehaltgrenze, werden diese für den Versicherer überhaupt nicht relevant. ${ }^{22}$ Dies trifft für das Arbeitslosigkeitsrisiko allerdings kaum zu, da

19 So schreibt Schönbäck (1988: 56): „Die große Zahl und Unterschiedlichkeit der Versicherten ermöglicht überdies die Bewältigung unerwartet großer sektoraler, regionaler oder sonstiger Instabilitäten des Arbeitsmarktes...".

20 Je stärker die Korrelation zwischen Risiken, desto weniger Risikotransformation kann ein Versicherungsunternehmen betreiben. Bei perfekt positiv korrelierten Risiken gibt es überhaupt keine Möglichkeit zur Risikotransformation. Versicherung bedeutet dann lediglich Risikotransfer (Meyer 1997: 19).

21 Unter diesem Begriff werden unterschiedliche Selbstbehaltsregelungen subsumiert (Helten/Karten 1984: 234 ff.).

22 Hartmann (1998: 133) führt als Beispiel die Sturmschäden von 1990 auf. Hierbei lag der durchschnittliche Einzelschaden bei 1000 DM. Eine absolute Abzugsfranchise in Höhe von 1000 DM hätte die Versicherungsunternehmen somit von einem Großteil der Einzelschäden befreit. 
bei einer Arbeitslosenversicherung die Vereinbarung einer absoluten Abzugsfranchise nur in sehr geringem Maße sinnvoll ist. ${ }^{23}$ So muss der Versicherer im Arbeitslosigkeitsfall fast immer leisten. Insofern ist die Wirkung großer Wirtschaftsrisiken - nämlich das Auftreten massenhafter Schäden auf das Versicherungsunternehmen unabhängig vom durchschnittlichen Deckungsgrad.

Bevor auf die einzelnen risikopolitischen Instrumente eingegangen wird, soll noch kurz die in Deutschland vorgeschriebene Spartentrennung ( 88 Abs. la VAG) erwähnt werden. Einzelne Versicherungszweige (Lebens-, Kranken- sowie Kompositversicherung) müssen demnach vollkommen getrennt betrieben werden. Dies soll die Transparenz erhöhen und dient letztlich dem Schutz des Versicherungsnehmers. Bereits 1972 hat Karten (1972: 193 f.) gefordert, dass sich die Trennung in einzelne Sparten nicht auf den Risikoausgleich beziehen dürfe. Eine dahingehende Lockerung der Spartentrennung würde ein erweitertes Diversifizierungspotential erschließen (Zweifel 1996: 262), welches zur Deckung der Abhängigkeit der Arbeitslosigkeitsrisiken genutzt werden könnte.

Neben all diesen einschränkenden Anmerkungen können zahlreiche Instrumente der modernen Versicherungstechnik aufgezeigt werden, mithilfe derer stochastisch abhängige Risiken versicherbar sind. Diese Elemente der Risikopolitik können auch kombiniert eingesetzt werden, wodurch der Versicherer spezifische Schwächen einzelner Instrumente gezielt ausgleichen kann: ${ }^{24}$

- Schwankungsfonds/Ausgleich über die Zeit

- Prämienanpassungen

- Kumulrelevante Risikomerkmale

- Festlegung einschränkender Versicherungsbedingungen

- Rückversicherung

- Alternativer Risikotransfer

${ }^{23}$ Eine absolute Abzugsfranchise im Fall der Arbeitslosigkeit würde bedeuten, dass erst $\mathrm{ab}$ einer gewissen Arbeitslosigkeitsdauer Versicherungsleistungen fällig werden (Karenzzeit). Eine relative Abzugsfranchise, welche gleichbedeutend mit einem Deckungsgrad kleiner 100 Prozent ist, wird wohl stets Bestandteil des Versicherungsvertrages sein. Der Versicherer leistet dann in jedem Schadenfall, allerdings ist die Versicherungsleistung kleiner als der entstandene (monetäre) Schaden.

${ }^{24}$ Für einen Überblick siehe Tabelle 1. 
Versicherungsunternehmen können über gesonderte Sicherheitszuschlä$\mathrm{ge}^{25}$ langfristige Reserven, sog. Schwankungsfonds, bilden (Hartmann 1998: 133). Durch Rückgriff auf diese Reserven können konjunkturelle Schwankungen, die das individuelle Arbeitslosigkeitsrisiko ceteris paribus erhöhen, abgefangen werden. ${ }^{26}$ Problematisch ist die Aufbaufinanzierung eines solchen Schwankungsfonds. Hierbei hätten die ersten Versichertenkohorten die größte Last zu tragen (Mooij 2004: 8). Zusätzlich ist davon auszugehen, dass bestimme Risikogruppen besonders stark von den Sicherheitszuschlägen betroffen wären. Dem Äquivalenzprinzip entsprechend würden Versicherungsunternehmen gerade bei jenen Versicherten besonders hohe Sicherheitszuschläge berechnen, die sehr stark von konjunkturellen Entwicklungen betroffen sind (Hartmann 1998: 180). Hier könnten allerdings gesetzliche Regelungen eine Umlage des konjunkturellen Risikos auf alle Versicherungsnehmer vorschreiben. Auch das Auftreten unsystematischer, asymmetrischer Konjunkturzyklen kann zu Problemen bei der Steuerung und richtigen Bemessung der Schwankungsfonds führen (Eekhoff 1996: 155 sowie Hartmann 1998: 180). Auch wenn sich konjunkturelle Schwankungen nach keinem Muster verhalten, ist ein Ausgleich über die Zeit funktionsfähig, sofern die einzelnen Konjunkturzyklen als unabhängige Zufallsereignisse interpretierbar sind. Helten/Karten (1984: 180) machen deutlich, dass ein Ausgleich über die Zeit in unstabilen Epochen schwierig, durch geschickte Transformation der Störungen jedoch dennoch möglich ist. Schließlich stellt die steigende Sockelarbeitslosigkeit, wie sie in Deutschland seit Jahren zu beobachten ist, ein Problem für die Wirksamkeit von Schwankungsfonds dar. Dem wäre nur durch eine Trendanpassung des Sicherheitszuschlags entgegen zu wirken.

Zusätzlich sei an dieser Stelle noch eine Bemerkung zum allgemeinen Sicherheitszuschlag als Bestandteil der Bruttorisikoprämie gemacht. Um langfristig zu überleben, müssen private Versicherungsunternehmen einen Sicherheitszuschlag auf die Nettorisikoprämie aufschlagen. Bei "richtiger“ Kalkulation bleibt dieser im Mittel übrig. Da der Sicherheitszuschlag nicht

25 Derartige Sicherheitszuschläge können aus der Varianz des Schadenerwartungswertes in Abhängigkeit konjunktureller Schwankungen berechnet werden. Sie sind sowohl für das gesamte Versichertenkollektiv als auch für Risikogruppen kalkulierbar.

${ }^{26}$ So sieht auch Eekhoff (1996: 155) das konjunkturelle Risiko als teilweise zeitlich ausgleichbar. 
dem Versicherungsunternehmen zugerechnet werden darf (Meyer 1997: 23) könnte er zum Aufbau eines Sicherheitskapitalstocks genutzt werden (Berliner 1982: 68).

Beenstock (1986: 11) empfiehlt bei steigenden Arbeitslosenquoten die Prämien entsprechend zu erhöhen und somit das Kumulrisiko auf die aktuelle Versichertengemeinschaft umzulegen. Als Vergleich führt er dabei die gestiegenen Prämien für Hausratversicherungen aufgrund erhöhter Einbruchraten an. Tatsächlich sind Prämienanpassungen durch gestiegene Schadenerwartungswerte in der Versicherungswirtschaft üblich. So sind beispielsweise Prämienerhöhungen aufgrund gestiegener Kopfschäden in der privaten Krankenversicherung durchaus zulässig (Meyer 1997: 21). Hartmann (1998: 180) merkt hierzu an, dass diese Vorgehensweise in Bezug auf eine private Arbeitslosenversicherung eine prozyklische Wirkung entfacht. Somit würden konjunkturelle Schwankungen in ihrer Wirkung noch verstärkt. Dem ist allerdings entgegenzusetzen, dass Prämienanpassungen stets zeitverzögert durchgeführt werden. Insofern ist nicht zwingend davon auszugehen, dass allgemeine Prämienerhöhungen aufgrund einer konjunkturellen Krise prozyklisch wirken.

Eine weitere Möglichkeit Kumulrisiken zu versichern sehen Glismann/ Schrader (2005: 68) in der Berücksichtigung kumulrelevanter Risikomerkmale bei der Prämienkalkulation. Die beiden Autoren schlagen vor, strukturelle Risiken in das individuelle Risikoprofil eines Versicherungsnehmers mit aufzunehmen. Zu denken ist hierbei insbesondere an Merkmale des Arbeitgebers, wie Branche oder Unternehmensgröße sowie regionale Gegebenheiten. Bestimmte Risikogruppen wären hierdurch stärker an der Finanzierung des Kumulrisikos beteiligt als andere. Damit die Aufnahme kumulrelevanter Risikomerkmale in die Prämienkalkulation überhaupt Sinn macht, muss dass Kumulrisiko ausreichend prognostizierbar sein. Glismann/Schrader (2005: 25) schreiben hierzu: „Um wie viel daher die Versicherungsprämien dieser Gruppen über dem Durchschnitt liegen müssten, dürfte sich freilich erst im Prozess des „Versuchens und Irrens" herausstellen." Ein "Prozess des Versuchens und Irrens" kann sich nur dann iterativ dem wahren Wert annähern, wenn letzterer fest gegeben ist oder sich nach einem festen Muster entwickelt. Nur sofern dies für konjunkturelle und strukturelle Entwicklungen zutrifft, stellen kumulrelevante Risikomerkmale eine Möglichkeit zur Diversifizierung des Arbeitslosigkeits- 
risikos dar. Änderungen im konjunkturellen und strukturellen Risiko können damit jedoch nicht gedeckt werden.

Zentrales Element einer privaten Versicherung ist die genaue Ausgestaltung des Versicherungsvertrags. Hierüber hat ein Versicherer die Möglichkeit bestimmte Leistungsfälle auszuschließen und dadurch Risiken vorzuselektieren. Dies kann auch genutzt werden, um Kumulschäden zu minimieren. So könnte ein Versicherungsvertrag derart ausgestaltet werden, dass eine bestimmte minimale Vorbeschäftigungsdauer (z. B. 24 Monate in den letzten drei Jahren) erfüllt sein muss, damit bei Eintritt einer Arbeitslosigkeit Anspruch auf die Versicherungsleistung besteht. Personen, die die minimale Vorbeschäftigungsdauer nicht erfüllen, würden somit nicht zum versicherten Personenkreis zählen. ${ }^{27}$ Außerdem können eine maximale Bezugsdauer, Karenzzeiten sowie Obliegenheiten für Versicherungsnehmer und bestimmte Leistungsausschlüsse festgelegt werden, wodurch potentielle Schadenfälle weiter eingegrenzt würden. Berufsanfänger, Langzeitarbeitslose sowie Personen, die in sehr kurzen Abständen mehrfach von Arbeitslosigkeit betroffen sind, wären dann z. B. nicht versichert. Wie bereits heute praktiziert, wäre hierfür eine staatliche Grundsicherung verantwortlich. Die genaue Ausgestaltung des Versicherungsvertrags kann die Wirkung stochastisch abhängiger Einzelrisiken sowie steigender Sockelarbeitslosigkeit abschwächen. Die Struktur des Problems bleibt freilich bestehen.

Ein sehr wirkungsvolles Instrument um Kumulrisiken zu beherrschen ist die Rückversicherung. Hierbei wird ein Erstversicherer (Zedent) für bezahlte Schäden von einem Rückversicherer (Zessionar) entschädigt und erlangt somit eigenen Versicherungsschutz für das übernommene Schadenpotential (Liebwein 2004: 2). Rückversicherung ist also die Versicherung der Versicherer (Swiss Re 2002: 9). Der eigentliche (primäre) Versicherungsnehmer hat keinerlei Ansprüche gegenüber dem Rückversicherer. Ein Zedent hat üblicherweise mehrere Rückversicherungspartner und auch Zessionare versichern sich ihrerseits rück (Retrozession). Auf diese Weise

27 Die Arbeitslosenzusatzversicherung, die 1996 von der Volksfürsorge auf den Markt gebracht wurde, hatte mit einer minimalen Vollzeit-Vorbeschäftigungsdauer beim selben Arbeitgeber von 3 Jahren sehr restriktive Leistungsbedingungen festgesetzt, wodurch die Risiken sehr stark vorselektiert wurden (Palan 1996: 92). 
kommt es zu einer „Atomisierung“ des Risikos (Pfeiffer 1999: 72), wodurch auch Großrisiken wie z. B. Kumule versichert werden können. Ein weiterer Grund, warum Rückversicherung ein essentieller Bestandteil der Kumulkontrolle ist, besteht in der Diversifizierungskapazität von Rückversicherungsunternehmen. So decken Rückversicherer unterschiedlichste Risiken von Erstversicherern auf der ganzen Welt (Swiss Re 2005: 31 f.).

Unter den verschiedenen Rückversicherungsformen (Pfeifer 1999: 42 ff.) kommt bei der Versicherung des Arbeitslosigkeitsrisikos die nicht-proportionale Rückversicherung und hierbei speziell die Kumulschadenexzedentenrückversicherung sowie die Jahresüberschadenrückversicherung (stop loss) in Betracht. Im ersten Fall wird die Summe aller Schäden eines Schadenereignisses rückversichert, sofern der Selbstbehalt (Priorität) des Erstversicherers überschritten ist. ${ }^{28}$ Von zentraler Bedeutung ist hierbei die präzise Definition des Schadenereignisses nach Ursache und zeitlichem sowie räumlichem Zusammenhang (Liebwein 2000: 162). Bei Konjunkturschwankungen als Ursache für Kumulschäden in der Arbeitslosenversicherung sollte dies, durch die präzisen Aufzeichnungen der wirtschaftlichen Aktivitäten seit vielen Jahrzehnten, kein Problem darstellen. Dies gilt auch für die Kumulkontrolle, die zur Identifikation von Kumulschäden und zur Festlegung von Priorität und Haftstrecke ${ }^{29}$ einem Rückversicherungsvertrag vorgeschaltet ist. Bei einem Kumulschadenexzedent muss der Erstversicherer den Schaden des gesamten Versichertenkollektivs nur bis zur Priorität tragen. Was darüber hinausgeht wird vom Rückversicherer geleistet. Diese Form der Rückversicherung wird häufig in Versicherungszweigen angewendet, in denen tendenziell geringe Einzelschäden auftreten, wie z. B. in der Allgemein Haftpflicht- und Kraftfahrt-Haftpflichtversicherung. Im Bereich der Lebensversicherung beispielsweise bietet ein Kumulschadenexzedent Schutz für den Fall, dass mehrere Personen durch ein Unfallereignis oder eine Naturkatastrophe ums Leben kommen (Pfeiffer 1999: 57). Eine Stop Loss Rückversicherung leistet, falls in einem Versicherungszweig die jährliche Summe aller Schäden die Priorität des Zeden-

${ }^{28}$ Hierbei wird eine Mindestzahl von Versicherungsnehmern festgelegt, die von diesem Schadenereignis betroffen sein muss. Geschieht dies nicht, so spricht man von einer Ereignisschadenexzedentenrückversicherung (Pfeiffer 1999: 55).

29 Darunter versteht man den Teil der Schadenverteilung, für den der Rückversicherer Haftung übernimmt. 
ten übersteigt. Hierbei spielt es keine Rolle, ob kleine und mittlere Schäden gehäuft auftreten oder ob hohe Einzelschäden anfallen. Die Priorität wird meist als Anteil der Bruttobeitragseinnahmen des Zedenten festgelegt, wobei darauf zu achten ist, dass Letztgenannter keinen Gewinn aus der Rückversicherung erzielen kann. Bei Inanspruchnahme einer Rückversicherungsleistung muss der Zedent stets einen Verlust erleidet, ansonsten besteht eine enorme Gefahr des Moral Hazard. Nicht-proportionale Rückversicherungsprodukte sind meist sehr teuer, da das Rückversicherungsunternehmen enorme Sicherheitszuschläge kalkulieren muss. Ursächlich hierfür sind fehlende Erfahrungswerte bezüglich der Schadenverteilung, die der Rückversicherer in Deckung nimmt. Im Zusammenhang mit gehäuft auftretender Arbeitslosigkeit ist die Datenlage jedoch sehr gut. So werden Arbeitmarktdaten bereits seit vielen J ahrzehnten erhoben. Insofern könnte eine Rückversicherung im Bereich des Arbeitslosigkeitsrisikos vergleichsweise günstig angeboten werden.

Meist wird die nichtproportionale mit einer proportionalen Rückversicherung kombiniert, so dass ein Rückversicherungsprogramm entsteht (Liebwein 2004: 23). ${ }^{30}$ Der isolierte Einsatz von proportionaler Rückversicherung ( $v$. a. die Quotenrückversicherung) bietet gegen Kumulschäden nur insofern Schutz, als der Zessionar an jedem Einzelschaden mitbeteiligt ist. Insofern wird die Wirkung eines Kumuls für den Zedenten abgeschwächt, die versicherungstechnische Problematik bleibt jedoch bestehen (Liebwein 2004: 14). Bei einer Rückversicherung des Arbeitslosigkeitsrisikos bezieht sich die Priorität sinnvollerweise auf die Schadensumme innerhalb eines definierten Zeitraums und wird entweder als Betrag oder als Anteil der Bruttobeitragseinnahmen des Zedenten festgelegt. Dann ist das Risiko unvorhersehbarer, gehäuft auftretender Arbeitslosigkeit vom Rückversicherer zu tragen. Darüber hinaus kann in Erwägung gezogen werden, den Staat als eine Art „Rückversicherer in letzter Instanz" einzusetzen. Bei der Versicherung von Terrorrisiken geschieht dies in Deutschland bereits heute schon (Nell/Richter 2003: $331 \mathrm{ff}$.).

30 Bei der proportionalen Rückversicherung wird das Risiko pro versicherungstechnischer Einheit zwischen Zedent und Zessionar nach einem festen Prozentsatz aufgeteilt. Dies gilt sowohl für Prämien als auch für Schäden (Pfeiffer 1999: 42). 
Seit etwa Anfang der 1990er Jahre wird neben der traditionellen Rückversicherung auch Risikotransfer außerhalb des Versicherungsmarktes betrieben. Bei diesen - unter dem Begriff "Alternativer Risikotransfer" subsumierten - Instrumenten ${ }^{31}$ wird der Kapitalmarkt als Risikoträger genutzt. Mit geschätzten 900 Mrd. US \$ täglichem Handelsvolumen sind die weltweiten Kapitalmärkte rund hundertmal größer als der Versicherungsmarkt und können damit bedeutend mehr Risiken und vor allem bisher nicht versicherbare Risiken absorbieren (Huber 2001: 12). Gegenwärtig wird alternativer Risikotransfer vor allem genutzt, um Naturkatastrophen zu versichern. Bei der Rückversicherung des Arbeitslosigkeitsrisikos kann der Einsatz von Kapitalmärkten als Risikoträger, (unerwartet) gehäuft auftretende Arbeitslosigkeit aufgrund wirtschaftlicher Schwankungen gegenfinanzieren. Bronars (1985) kann mit amerikanischen Daten von 1956-82 zeigen, dass die Ausgaben der Arbeitslosenversicherung positiv korreliert sind mit den Renditen am Aktienmarkt. Durch Hedging des durchschnittlichen Arbeitslosigkeitsrisikos mit einem antizyklisch korrelierten Finanzmarktinstrument könnte auch das Kumulrisiko "Arbeitslosigkeit“ versichert werden. Dies bestätigt auch die Arbeit von Haley (1990). Hier wird gezeigt, dass durch Hedging mit US T-Bill Futures die Varianz einer notwendigen Reserve für eine Arbeitslosenversicherung halbiert werden kann (Haley 1990: 75). Dadurch wird die Versicherung von Arbeitslosigkeit auch im Konjunkturverlauf möglich. Sofern die zur Absicherung von Arbeitslosigkeit notwendige Kapitalmenge hinreichend groß ist, könnte es zu einer Rückkopplung zwischen Kapital- und Versicherungsmarkt kommen (Hartmann 1998: 179), was die Funktionsfähigkeit eines alternativen Risikotransfers beeinträchtigen würde. Aufgrund der enormen Diversifizierungsmöglichkeiten auf den weltweiten Kapitalmärkten und der nationalen Begrenzung einer Arbeitslosenversicherung ist davon jedoch nicht auszugehen.

${ }^{31}$ Für einen ausführlichen Überblick über die verschiedenen Instrumente des alternativen Risikotransfers siehe Liebwein (2000: 365 ff.), Swiss Re 2003, Swiss Re 1999. 
Tabelle 1: Risikopolitische Instrumente im Überblick

\begin{tabular}{|c|c|c|}
\hline Instrument & $\begin{array}{c}\text { (hautsächlicher) Träger } \\
\text { des Kumulrisikos }\end{array}$ & Besonderheiten \\
\hline Schwankungsfonds & Versicherungsunternehmen & $\begin{array}{l}\text { Aufbaufinanzierung } \\
\text { unsystematische/ asymmetri- } \\
\text { sche Schocks sind nicht ge- } \\
\text { deckt }\end{array}$ \\
\hline Prämienanpassung & $\begin{array}{l}\text { aktuelle } \\
\text { Versichertengemeinschaft }\end{array}$ & ev. prozyklische Wirkung \\
\hline $\begin{array}{l}\text { Kumulrelevante } \\
\text { Risikomerkmale }\end{array}$ & einzelne Risikogruppen & $\begin{array}{l}\text { unsystematische/ } \\
\text { asymmetrische Schocks sind } \\
\text { nicht gedeckt } \\
\text { (teilweise) verursachungsge- } \\
\text { rechte Zuordnung der } \\
\text { Kumulkosten }\end{array}$ \\
\hline $\begin{array}{l}\text { Einschränkende } \\
\text { Versicherungsbedingungen }\end{array}$ & Versicherungsunternehmen & $\begin{array}{l}\text { Kumulrisiko bleibt für die } \\
\text { Versicherungsunternehmen in } \\
\text { seiner Struktur bestehen }\end{array}$ \\
\hline Rückversicherung & Rückversicherer & $\begin{array}{l}\text { Lange Tradition und damit } \\
\text { zahlreiche zur Verfügung } \\
\text { stehende Produkte }\end{array}$ \\
\hline Alternativer Risikotransfer & Kapitalmarkt & $\begin{array}{l}\text { Enormes Potential, aber } \\
\text { vergleichsweise neues } \\
\text { Instrument } \\
\text { ev. Rückkopplung zwischen } \\
\text { Kapital- und Versicherungs- } \\
\text { märkten }\end{array}$ \\
\hline
\end{tabular}

Quelle: Eigene Darstellung

Als Zwischenfazit lässt sich festhalten, dass dem Versicherungsgewerbe heute ein umfassendes Instrumentarium zu Verfügung steht, unter dessen Einsatz auch stochastisch abhängige Einzelrisiken, wie Arbeitslosigkeit, versichert werden können. Der kombinierte Einsatz von Rückversicherung und alternativem Risikotransfer kann hierbei eine entscheidende Rolle übernehmen. Die Weiterentwicklungen in der Versicherungstechnik, die in den letzten Jahren auch die Versicherung gegen Naturkatastrophen ermöglicht hat, lässt kaum Zweifel daran, dass auch das Kumulrisiko von Arbeitslosigkeit versichert werden kann. ${ }^{32}$ Lediglich große Wirtschaftskrisen stellen ein echtes Problem für eine private Arbeitslosenversicherung

32 Auch Chiu und Karni (1998: 819) bestätigen, dass das Kumulrisiko von Arbeitslosigkeit einer privaten Versicherung nicht im Wege steht. Eine staatliche Arbeitslosenversicherung würde den beiden Autoren zufolge, eher zu Ineffizienten führen, da Kumulrisiken hierbei über das Steuersystem gedeckt werden. 
dar. Dies gilt allerdings für den gesamten privaten Finanzsektor einer Volkswirtschaft. Private Lebens- und Krankenversicherer wären mit großen Wirtschaftskrisen ähnlich überfordert wie eine Arbeitslosenversicherung.

\subsection{Probleme unvollständiger I nformation}

\subsubsection{Die individuellen Wahrscheinlichkeiten arbeitslos zu werden bzw. zu bleiben sind nicht hinreichend bekannt}

Versicherungsunternehmen können nur dann langfristig ihr Überleben sichern, wenn die hypothetische Schadenverteilung des gesamten Versichertenkollektivs nicht zu stark von der tatsächlichen Schadenverteilung abweicht. Letztere ergibt sich immer erst im Nachhinein und ist zum Zeitpunkt der Prämienkalkulation niemals bekannt. Für kaum einen Schadenfall gibt es längere Zeitreihen und besserer Statistiken als für Arbeitslosigkeit. Insofern ist die Gesamtschadenverteilung für Arbeitslosigkeit sehr gut prognostizierbar und es ist davon auszugehen, dass die Abweichung zwischen einer hypothetischen und tatsächlichen Gesamtschadenverteilung vergleichsweise gering ausfällt. Vor diesem Hintergrund scheinen die folgenden Ausführungen von Berthold/von Berchem (2002: 60) wenig stichhaltig: "Sowohl die Wahrscheinlichkeitsverteilung des Eintritts der Arbeitslosigkeit als auch die zu erwartende Höhe des resultierenden Schadens muss als nicht hinlänglich bekannt erachtet werden. Für potentielle private Versicherer sind die Arbeitslosigkeitsrisiken daher kaum kalkulierbar, und sie würden ein solch unkalkulierbares Risiko nur bei derart exorbitant hohen Prämien eingehen wollen, dass für einen Großteil der Arbeitnehmer eine Versicherung nicht möglich oder nicht rational wäre." An diesem Zitat lässt sich unter anderem die weit verbreitete Vermengung von versicherungstechnischer und normativer Perspektive demonstrieren. Wie bereits erwähnt, stellt der Umstand, dass sich einige Personen keinen Versicherungsschutz leisten können, kein versicherungstechnisches Problem dar, sondern ist unter Verteilungs- und Teilhabegesichtpunkten zu bewerten. Das Argument, dass die individuellen Arbeitslosigkeitsrisiken nicht hinreichend bekannt seien, bezieht sich offenbar auf etwas vollkommen anderes als auf die grundsätzliche Schätzbarkeit der Gesamtschadenverteilung. Es geht vielmehr um die Anwendung des individuellen Äquivalenzprinzips und damit um die verursachungsgerechte Zurechnung des 
Erwartungsschadens des gesamten Kollektivs auf die einzelnen Versicherungsnehmer (Farny 2006: 67).

Damit Versicherungsunternehmen risikoäquivalente Prämien berechnen können, benötigen sie hinreichend genaue Informationen über Schadenerwartungswerte unterschiedlicher Risikogruppen. Die Ausdifferenzierung des Versichertenkollektivs in einzelne Risikogruppen ist dann ausreichend genau, wenn der einzelne Versicherungsnehmer seine individuelle Risikoposition innerhalb seiner Risikogruppe nicht mehr einschätzen kann (Glismann/Schrader 2005: 65). Er darf also keinen „spürbaren“ Informationsvorsprung vor seinem Versicherer haben. Der von zahlreichen Autoren geäußerte Zweifel an der Funktionsfähigkeit einer privaten Arbeitslosenversicherung kann in diesem Zusammenhang nur bedeuten, dass es Versicherungen nicht gelingt, die Prämien ausreichend genau zu differenzieren. Die Tatsache, dass sowohl Schadeneintritt als auch Schadenhöhe unsicher sind, ist dagegen kein Argument gegen eine private Versicherung. Wie Glismann/Schrader (2005: 69) richtig bemerken, ist Unsicherheit gerade die Voraussetzung für eine Versicherung.

Ist ein Versicherungsunternehmen nicht in der Lage, die Risiken ausreichend zu differenzieren, dann kann der einzelne Versicherungsnehmer die relative Höhe seines Schadenerwartungswerts innerhalb seiner Risikogruppe einordnen. Ein gutes Risiko - also ein Versicherungsnehmer mit relativ geringem Schadenerwartungswert - weiß dann, dass es ein gutes Risiko ist. Die für seine Risikogruppe kalkulierte Durchschnittsprämie erscheint ihm zu hoch und er wird sich deshalb entscheiden, keinen Versicherungsvertrag abzuschließen bzw. seinen bestehenden Vertrag zu kündigen. Übrig bleiben die schlechten Risiken. Um weiterhin den Schadenerwartungswert dieser Risikogruppe durch die Prämienzahlungen zu decken, muss das Versicherungsunternehmen die Prämie nun erhöhen. Unter den verbliebenen schlechten Risiken können erneut weniger schlechte und sehr schlechte getrennt werden. Wieder ist die kalkulierte Durchschnittsprämie für die weniger schlechten Risiken zu hoch. Sie werden die Versicherung verlassen, worauf das Versicherungsunternehmen abermals gezwungen ist, die Prämie nach oben anzupassen. Dieser Prozess setzt sich so lange fort, bis letztlich nur die schlechtesten Risiken bei der Versicherung verbleiben. Dieses von George Akerlof (1970) erstmals aufgezeigte Phänomen wird als adverse Selektion bezeichnet und hat seinen Ursprung 
in einer asymmetrischen Informationsverteilung zwischen Versicherungsnehmer und Versicherungsunternehmen. Es hat zur Folge, dass letztlich nur die schlechtesten Risiken einen Versicherungsschutz erhalten zu dementsprechend hohen Prämien.

Zahlreiche empirische Arbeiten haben bereits gezeigt, dass das Arbeitslosigkeitsrisiko hinreichend genau erfasst werden kann. ${ }^{33}$ Vor allem soziodemographische Merkmale spielen dabei eine entscheidende Rolle. Es gelingt jedoch niemals, alle relevanten Risikomerkmale zu berücksichtigen, weshalb sich die einzelnen Versicherungsnehmer auch innerhalb einer Risikogruppe in ihrer Risikostruktur stets unterscheiden. Um die Inhomogenitäten innerhalb einer Risikogruppe zu mildern, betreiben Versicherer sekundäre Prämiendifferenzierung (Erfahrungstarifierung). ${ }^{34} \mathrm{Im}$ Gegensatz zur primären Prämiendifferenzierung orientiert sich diese an den tatsächlich verursachten Schäden. Dies kann sowohl im Nachhinein in Form von Beitragsrückerstattungen geschehen, als auch innerhalb der Prämienkalkulation im Vorhinein. Die im letzteren Fall zum Einsatz kommenden mathematischen Methoden werden durch die so genannte Credibility-Theorie beschrieben. Die Grundformel des Credibility-Rating hat die Form $N R P_{j}=c\left(\frac{1}{n} \sum_{i=1}^{n} s_{j i}\right)+(1-c) N R P_{k}$, wobei $N R_{j}$ die Nettorisikoprämie des j-ten Versicherungsnehmers bezeichnet, $\mathrm{s}_{\mathrm{ji}}$ die Versicherungsleistungen für den j-ten Versicherungsnehmer im Jahr i kennzeichnet und $\mathrm{NRP}_{\mathrm{K}}$ die Nettorisikoprämie des Versicherten(teil)kollektivs beschreibt. Der Beobachtungszeitraum ist durch $\mathrm{n}$ gegeben. Je größer c (Credibility-Faktor), umso mehr Glaubwürdigkeit wird dem individuellen Schadenverlauf beigemessen (von Schaaffhausen 1989: 14). Die grundlegende Annahme ist, dass die vergangene Schadenentwicklung einer Person Informationen über zukünftig zu erwartende Schäden beinhaltet, welche mit den nicht berücksichtigten Risikomerkmalen korrelieren. Nimmt man also die vergangenen Schäden in die Prämienkalkulation mit auf, so greift man damit jene Risikomerkma-

33 Arulampalam/Stewart (1995), Bender et al. (2000), Biewen/Wilke (2005), Fieldhouse (1996), Hujer/Schneider (1996), Licht/Steiner (1991), Narendranathan/Stewart (1993), Uhlendorf (2003), um nur einige zu nennen.

34 Dionne/Lasserre (1985) können zeigen, dass durch Erfahrungstarifierung dem Problem der adversen Selektion begegnet werden kann und eine effiziente Versicherungslösung zustande kommt. 
le ab, die nicht direkt erfasst werden können. Im Falle von Arbeitslosigkeit ist hierbei z. B. an die Motivation und Einsatzbereitschaft einer Person zu denken. Der Einwand von Berthold/von Berchem (2004: 7), dass eine Kategorisierung der Risiken an der Unbeobachtbarkeit dieser Merkmale scheitert, ist insofern zu relativieren.

Betrachtet man die Ergebnisse einiger empirischer Arbeiten über den Einfluss früherer auf zukünftige Arbeitslosigkeit, so könnte es an dieser Stelle leicht zu Missverständnissen kommen. So zeigen z. B. Heckman/Borjas (1980), dass es keinerlei kausalen Zusammenhang zwischen Anzahl oder Dauer frührer Arbeitslosigkeitsepisoden und zukünftiger Arbeitslosigkeit gibt. Für die Prämienkalkulation einer Versicherung sind jedoch lediglich statistische Korrelationen von Bedeutung. Ob sich dahinter kausale Zusammenhänge verbergen, ist weitestgehend unerheblich. Dass eine Korrelation zwischen früherer und zukünftiger Arbeitslosigkeit besteht, konnten indes zahlreiche Untersuchungen belegen. ${ }^{35}$

Neben einer aktiven Prämiendifferenzierung seitens des Versicherers kommt es auch durch die individuelle Vertragswahl der Versicherungsnehmer (z. B. Höhe des Deckungsgrades) zu einer Selektion der Risiken (Selbstselektion) ${ }^{36}$ Hierdurch offenbart der einzelne Versicherungsnehmer teilweise seine Risikoposition, was der Versicherer zur Risikodifferenzierung nutzen kann (Hartmann 1998: 150 ff.). In diesem Zusammenhang konnten Rothschild/Stiglitz (1976) modelltheoretisch zeigen, dass unter bestimmten Voraussetzungen ${ }^{37}$ selbst dann ein Versicherungsschutz für gute Risiken möglich ist, wenn asymmetrische Information vorliegt und Versicherungsunternehmen keinerlei Möglichkeiten haben, diese abzumildern. Selbstselektion führt dazu, dass gute Risiken Teildeckung nachfra-

35 Siehe z. B. Arrow (1996), Corak (1993), Elias/Steiner (1998), Steiner (1990).

${ }^{36}$ Chiu/Karni (1998: 819) weisen auf die Wichtigkeit der Vertragswahl und damit der Selbstselektion hin. Aufgrund privater Informationen auf Seiten des Arbeitnehmers bezüglich individueller Freizeitpräferenzen kann es ansonsten zur Unversicherbarkeit des Arbeitslosigkeitsrisikos kommen.

37 Dies betrifft die Zusammensetzung des Versichertenkollektives. Gibt es relativ wenige gute Risiken, so kommt es zu einem trennenden Gleichgewicht, bei dem die schlechten Risiken Vollversicherung und die guten Risiken Teilversicherung erhalten (Rothschild/Stiglitz 1976: 637). 
gen, schlechte Risiken dagegen tendenziell höheren Deckungsschutz präferieren. ${ }^{38}$

Alles in allem zeigt sich, dass das Argument einer mangelnden Quantifizierbarkeit des Arbeitslosigkeitsrisikos nicht überzeugend ist. ${ }^{39}$ Zum einen besteht die Möglichkeit, individuelle Schadenerwartungswerte aus Arbeitslosigkeit hinreichend genau zu schätzen, zum anderen stehen den Versicherern durch Erfahrungstarifierung und vielfältige Vertragsgestaltung Mittel zur Verfügung, um Risiken ausreichend zu differenzieren.

\subsubsection{Moral Hazard}

Unter dem Begriff Moral Hazard versteht man das Nachlassen der Sorgfalt bei der Schadenverhütung bzw. das mutwillige Herbeiführen eines Schadenfalles allein aufgrund eines bestehenden Versicherungsschutzes. Ist ein Schaden bereits eingetreten, so bezeichnet ex post Moral Hazard ein Verhalten, das nicht auf Schadenminimierung sondern auf individuelle Nutzenmaximierung ausgerichtet ist. Moral Hazard entsteht dann, wenn der Versicherer das Verhalten des Versicherten sowie anderer schadenbeeinflussender Akteure nicht ausreichend kontrollieren bzw. sanktionieren kann (Stiglitz 1983: 5). Da eine vollständige Kontrolle jedoch niemals gelingt, ist Moral Hazard ein Problem, mit dem sich jede Versicherungsform grundsätzlich auseinandersetzen muss. Je schwieriger die Kontrolle und Sanktionierung für den Versicherer, desto schwerer wiegt die Moral Hazard Problematik. Vor allem bei neuen Versicherungsprodukten, bei denen es keine Erfahrungswerte gibt, kann das moralische Risiko schwerwiegende Folgen für den Versicherer haben (Mugler 1980: 77). So kann die Schadenverteilung durch Moral Hazard stark beeinflusst werden (Arrow 1971: 142), wobei die genaue Wirkung zum Zeitpunkt der Prämienkalkulation nicht abzusehen ist. Insofern kann es zu großen Abweichungen zwischen hypothetischer und tatsächlicher Schadenverteilung kommen, was letztlich das Überleben des Versicherers gefährdet. Eine private Arbeitslosenversicherung stellt insofern keine echte Produktinnovation dar, als be-

38 Barr (1992: 752) weist allerdings darauf hin, dass dieses separierende Gleichgewicht nicht effizient ist, da es guten Risiken nicht ermöglich, volle Deckung zu erhalten.

39 Dies bestätigt selbst Berthold (1988: 364) als Kritiker einer privaten Arbeitslosenversicherung. 
reits seit Langem eine staatliche Versicherung existiert, die auch mit dem Problem des Moral Hazard zu kämpfen hat. Somit sind in heutigen Schadendaten bereits die Wirkungen des Verhaltensrisikos teilweise enthalten.

Besonders problematisch ist der Umstand, dass das individuelle Verhaltensrisiko mit zunehmender Prämie ebenfalls wächst (Farny 2006: 34). Demnach kommt es zu einer Aufwärtsspirale zwischen moralischem Risiko und Prämie, was letztlich zur Unversicherbarkeit führt (Helten/Karten 1984: 135). So ist davon auszugehen, dass der einzelne Versicherungsnehmer sein individuelles Verhaltensrisiko besser einschätzen kann als der Versicherer und dass Moral Hazard ceteris paribus zu einer ungünstigeren Schadenverteilung und damit zu höheren Prämien führt. Unterscheidet man das Versichertenkollektiv vereinfachend in Personen, die Moral Hazard „betreiben“ und solche, die dies nicht tun, so erhalten im Endeffekt nur jene Personen einen Versicherungsschutz, die Moral Hazard „betreiben“. Alle übrigen Personen verlassen die Versicherung, da ihnen die kalkulierte Prämie zu hoch erscheint. Insofern kann moralisches Risiko den bereits beschriebenen Prozess der adversen Selektion einleiten. Kann der Versicherer das Verhaltensrisiko nicht hinreichend ausschließen, so ist das entsprechende Risiko nicht versicherbar (Faure 1995: 456).

Ein Charakteristikum der Arbeitslosenversicherung ist, dass zahlreiche Akteure identifiziert werden können, die potentiell einem Verhaltensrisiko unterliegen. Die Literatur unterscheidet hierbei folgende Hasardeure: ${ }^{40}$

- Versicherungsnehmer

- Arbeitgeber

40 Moral Hazard ist grundsätzlich zu trennen von negativen externen Effekten. Letzteres bezeichnet die nicht kompensierten Kosten einer Aktivität auf Dritte (Barr 2001: 15) und steht im Gegensatz zum moralischen Risiko nicht in systematischem Zusammenhang mit Versicherung. Externe Effekte verstärken sich auch nicht durch - von ihnen ausgelöste - Prämienerhöhungen. Lediglich neu auftretende externe Effekte, die nicht bereits in den zur Kalkulation verwendeten Daten enthalten sind, sind problematisch, da sie zu einer Änderung des Risikoursachensystems führen (Farny 2006: 90 f.). Dies ist allerdings ein globales Problem, das jeder Form von Versicherung stets droht (Klimawandel, zunehmende Verkehrsdichte, verstärkte soziale Konflikte, technischer Fortschritt, veränderte Gesetzgebung). Auch die Arbeitslosenversicherung bildet hier keine Ausnahme, sondern unterliegt ebenfalls einem ständigen Änderungsrisiko. Da es sich hierbei jedoch nicht um eine Besonderheit des Risikos „Arbeitslosigkeit“ handelt, werden externe Effekte im Folgenden nicht explizit thematisiert. Entgegen dem allgemeinen Vorgehen in der Literatur wird allerdings auf die Trennung zwischen Moral Hazard und externen Effekten besonders geachtet. 
- Tarifvertragsparteien

- Versicherungsunternehmen

- politische Entscheidungsträger

Auf Seiten des Versicherungsnehmers kann die Versicherung des Arbeitslosigkeitsrisikos zu einer Verhaltensänderung dahingehend führen, dass der Schadenfall häufiger eintritt (Grubel 1995: 6 f.). Gründe hierfür können z. B. in nachlassendem Arbeitseifer liegen. Außerdem kann sich Moral Hazard dadurch äußern, dass ein bereits eingetretener Schaden höher ausfällt. Dieses ex post moralische Risiko kann bei arbeitslosen Versicherungsnehmern in Form von mangelnder Mobilität, unterlassener Aus- und Weiterbildung sowie höherer Forderungen gegenüber dem Arbeitgeber (Berthold/von Berchem 2004: 8) auftreten, was letztlich zu längeren Arbeitslosigkeitsdauern führt. Ob diese versicherungsinduzierten Verhaltensänderungen beim Versicherungsnehmer tatsächlich in großem Maßstab zu beobachten sind, ist allerdings fraglich. ${ }^{41}$ So umfasst der Schaden aus Arbeitslosigkeit eben nicht nur das entgangene Arbeitnehmerentgelt, sondern auch psychische Kosten, die durch eine empfundene gesellschaftliche und soziale Ausgrenzung entstehen (Sesselmeier et al. 2006: 26). Diese außervertraglichen Hemmnisse führen dazu, dass der tatsächliche Schaden durch eine Arbeitslosenversicherung niemals zu 100 Prozent gedeckt ist. Darüber hinaus wird ein Versicherer auch den monetären Schaden nicht vollständig in Deckung nehmen, sondern Verträge mit unterschiedlichen Deckungsgraden anbieten. Durch die Wahl eines bestimmten Vertrages gibt der Versicherungsnehmer außerdem wichtige Informationen über sein potentielles Verhaltensrisiko preis, die vom Versicherer zur Risikoselektion genutzt werden können. Ganz allgemein stellt die Deckungsabgrenzung via Franchisen und zeitlicher Leistungsbegrenzung für den Versicherer ein starkes Instrument zur Eindämmung von Moral Hazard seitens des Versicherungsnehmers dar.

${ }^{41}$ Es existieren zahlreiche mikroökonometrische Arbeiten, die das moralische Risiko in der deutschen Arbeitslosenversicherung untersucht haben (Glismann/Schrader 2005: $14 \mathrm{ff}$. sowie $211 \mathrm{ff}$.). Im Zentrum steht hierbei jeweils der Zusammenhang zwischen Lohnersatzrate bzw. maximaler Bezugsdauer und Höhe sowie Dauer der Arbeitslosigkeit. Bewertet man all diese Studien in ihrer Summe, so zeigt sich vor allem ein Zusammenhang zwischen maximaler Bezugsdauer und Dauer der Arbeitslosigkeit (Sachverständigenrat 2003: 395). 
Ein Versicherungsvertrag kann natürlich auch im Zeitverlauf sinkende Versicherungsleistungen festlegen, was in theoretischen Arbeiten bereits als sinnvoll herausgearbeitet wurde (Hopenhayn/Nicolini 1997 sowie Shavell/ Weiss 1979) und unter anderem auch vom Sachverständigenrat (2003: 397 f.) gefordert wird. Zusätzlich hat der Versicherer die Möglichkeit, bestimmte Obliegenheiten im Versicherungsvertrag festzulegen, die den Versicherungsnehmer zu einem schadenvermeidenden sowie -minimierenden Verhalten verpflichten. Über Prämienvergünstigungen kann er auch Anreize für ein erwünschtes Verhalten setzen (Grubel 1995: 10). So könnte beispielsweise die Teilnahme an Fort- und Weiterbildungsmaßnahmen prämienwirksam gefördert werden oder Versicherungsnehmer dazu verpflichtet werden, im Arbeitslosigkeitsfall an bestimmten Maßnahmen, wie z. B. Bewerbungstrainings, teilzunehmen. Letztlich können auch BonusMalus-Systeme im Zuge einer Erfahrungstarifierung das moralische Risiko eindämmen (Hartmann 1998: 171). Dies zeigt sich anhand des Systems der Schadenfreiheitsklassen in der Kfz-Versicherung. Ganz ähnlich könnte auch eine private Arbeitslosenversicherung den vergangenen Schadenverlauf in der Prämiekalkulation berücksichtigen und hierdurch Moral Hazard von Seiten des Versicherungsnehmers reduzieren. Dies wird im Übrigen auch durch theoretische Arbeiten zum optimalen Versicherungsvertrag in der Arbeitslosenversicherung gestützt (Hopenhayn/Nicolini 1997). ${ }^{42}$ Wie auch Glismann/Schrader (2005: 61) sowie Berthold (1988: 362) in ihren Analysen feststellen, scheint Moral Hazard beim Versicherungsnehmer kein Problem für die private Versicherbarkeit des Arbeitslosigkeitsrisikos zu sein. Es ist vielmehr davon auszugehen, dass der private Sektor - viel eher als der Staat - in der Lage ist, diesem moralischen Risiko entgegenzuwirken (Schäfer 2006: 25 sowie Berthold/Külp 1987: 39) und damit letztlich die Wohlfahrt zu erhöhen (Grubel 1995: 10).

Nach der vorherrschenden Meinung in der Literatur betreiben Arbeitgeber Moral Hazard, indem sie ihr Entlassungsverhalten anpassen. ${ }^{43}$ Demnach führt die Existenz einer Arbeitslosenversicherung dazu, dass Unternehmen schneller temporäre Entlassungen vornehmen (Mooij 2004: 9), weil sie die

42 Schäfer (2003a: 47) weist allerdings darauf hin, dass durch eine Erfahrungstarifierung der Anspruchlohn eines Arbeitslosen steigt.

43 Z. B. Berthold/von Berchem (2004: 8), Glismann/Schrader (2005: 62), Sachverständigenrat (2003: 393), Sesselmeier et al. (2006: 19). 
anfallenden Entlassungskosten auf die Arbeitslosenversicherung abschieben können (Berthold 1988: 362). Für die USA der 70er Jahre beispielsweise hat Feldstein (1978) ermittelt, dass etwa 75 Prozent aller Entlassungen in der verarbeitenden Industrie temporärer Natur sind, die freigesetzten Arbeitnehmer also zu einem späteren Zeitpunkt wieder bei demselben Arbeitgeber beschäftigt werden. Somit ist es nicht verwunderlich, dass in den Vereinigten Staaten das System des "experience rating Anwendung findet, wonach der Arbeitgeber entsprechend seines individuellen Entlassungsverhaltens an der Finanzierung der Arbeitslosenversicherung beteiligt ist. Grundsätzlich stellt sich jedoch die Frage, ob Arbeitgeber eher temporäre Entlassungen vornehmen, allein weil eine Arbeitslosenversicherung existiert. Nur dann kann von arbeitgeberseitigem Moral Hazard gesprochen werden. Andernfalls liegen lediglich negative externe Effekte vor, wie sie im privaten Versicherungsbereich immer wieder zu beobachten sind.

Die Arbeitsnachfrage der Unternehmen orientiert sich an einem rationalen Kalkül. Mitarbeiter werden nur dann entlassen, wenn dies für den Arbeitgeber rational im Sinne der Gewinn- bzw. Nutzenmaximierung ist. In einer Phase der Unterauslastung gibt es Mitarbeiter, für die die Lohnkosten den Beitrag zur Produktivität übersteigen. Dies stellt quasi die Kosten einer Weiterbeschäftigung dar. Die Kosten einer temporären Entlassung liegen in einer motivations- und produktivitätsmindernden Wirkung auf alle Mitarbeiter, in der erneuten Personalsuche und -auswahl, dem Risiko einer späteren Stellenfehlbesetzung sowie im Abgang allgemeinen und vor allem firmenspezifischen Humankapitals (Holz/Hauser 2000: 16). Vor allem bei mittelständischen Betrieben kann zusätzlich eine ausgeprägte soziale Verantwortung gegenüber den Mitarbeitern bestehen, die sich ebenfalls in den Kosten einer temporären Entlassung niederschlagen. Das (temporäre) Entlassungsverhalten des Arbeitgebers wird sich daran orientieren, ob die Kosten einer Weiterbeschäftigung oder einer temporären Entlassung überwiegen. Eine Arbeitslosenversicherung trägt nun dazu bei, dass die Kosten der Entlassung gesenkt werden. Man kann quasi von einer impliziten Vereinbarung zwischen Arbeitgeber und Arbeitnehmer ausgehen (Berthold/Külp 1987: 46), wonach in einer Phase der Unterauslastung Mitarbeiter in der Arbeitslosenversicherung lediglich "geparkt" werden. Es ist allerdings zu berücksichtigen, dass insbesondere gering qualifizierte Mit- 
arbeiter aus speziellen Branchen von temporären Entlassungen betroffen sind (Mavromaras/Rudolph 1995: 178), für die die oben genannten Entlassungskosten vergleichsweise gering ausfallen. Für dieses Arbeitnehmersegment scheint eine effektive Senkung der Entlassungskosten durch eine Arbeitslosenversicherung somit fraglich. Folglich kann hierbei auch nicht eindeutig von Moral Hazard gesprochen werden. Mit anderen Worten: Denjenigen Arbeitnehmern, die hauptsächlich von temporären Entlassungen betroffen sind, wird sowohl mit als auch ohne Arbeitslosenversicherung gekündigt.

Allerdings werden die Entlassungskosten, die durch die soziale Verantwortung des Arbeitgebers gegenüber seinen Mitarbeitern entstehen, durch eine Arbeitslosenversicherung merklich reduziert. So weiß der Arbeitgeber seinen Mitarbeiter für die Dauer der temporären Entlassung gut versorgt. Der Arbeitgeber unterliegt lediglich an dieser Stelle einem Verhaltensrisiko. Jedoch müssten jene Arbeitnehmer, die von temporären Entlassungen betroffen sind, in einem System privater Arbeitslosenversicherung Prämienerhöhungen erleiden, da sich die Prämienkalkulation am vergangenen Schadenverlauf orientieren würde. Diesen Umstand müsste der Arbeitgeber dann in sein Entlassungskalkül mit aufnehmen. Seine soziale Verantwortung gegenüber seinem Mitarbeiter würde dann eher zur Weiterbeschäftigung führen und damit die Einführung von Jahresarbeitszeitkonten, eine langfristig orientierte Personalpolitik sowie den Einsatz von Zeitarbeit und befristeter Beschäftigung begünstigen. Ein System privater Arbeitslosenversicherung, welches risikoäquivalente Prämien beim Arbeitnehmer veranschlagt, reduziert folglich arbeitgeberseitiges Moral Hazard.

Hinter dem in der Literatur beschriebenen Moral Hazard beim Arbeitgeber verbirgt sich zu einem Großteil ein Mitnahmeeffekt, der von arbeitsmarktpolitischen Instrumenten wie z. B. Einstellungszuschüsse für Geringqualifizierte noch verstärkt wird (Holz/Hauser 2000: 18). Es ist zu bezweifeln, dass arbeitgeberseitiges Moral Hazard in einer privaten Arbeitslosenversicherung tatsächlich in hinreichend großem Umfang zu beobachten sein wird (Holz/Hauser 2000: 16). ${ }^{44}$ Die Erwartung, wieder im alten Betrieb beschäftigt zu werden, beeinflusst wohl vielmehr das Suchverhalten der Ar-

${ }^{44}$ Schneider et al. (2004: 66 f.) verweisen hierzu auf den in Deutschland existierenden Kündigungsschutz, der temporären Entlassungen entgegensteht. 
beitlosen (Holz/Hauser 2000: 17) und ist insofern eher als Moral Hazard von Seiten des Versicherungsnehmers zu interpretieren. Eine verursachungsgerechte Beteiligung der Arbeitgeber an der Finanzierung der Arbeitslosenversicherung ist aus versicherungstechnischer Sicht folglich nicht notwendig. Natürlich würde ein „experience rating“ dennoch die Entlassungskosten erhöhen und damit die Anzahl temporärer Entlassungen reduzieren. Außerdem würde dadurch die bestehende Quersubventionierung zwischen einzelnen Wirtschaftzweigen (Genosko et al. 1999) aufgelöst und somit der strukturelle Wandel beschleunigt (Berthold/von Berchem 2002: 63). Allerdings kann eine am Entlassungsverhalten ausgerichtete arbeitgeberseitige Finanzierung der Arbeitslosenversicherung auch Wachstumshemmnisse beinhalten, da kleine und innovative Risikounternehmen dabei stärker belastet werden. Es ist weiterhin anzunehmen, dass eine gestiegene Beschäftigungsstabilität zu Lasten der Arbeitslosen geht, für die die Wiedereinstellungsmöglichkeiten geringer werden (Holz/Hauser 2000: 33 f.). Es gäbe dann zwar eine geringe Anzahl von Eintritten in Arbeitslosigkeit, die durchschnittliche Dauer einer Arbeitslosigkeitsepisode würde jedoch steigen (Holz/Hauser 2000: 58 f.). Letztlich muss offen bleiben, ob eine Beteiligung der Unternehmen an der Finanzierung der Arbeitslosenversicherung sinnvoll ist. Aus versicherungstechnischer Sicht scheint sie zumindest nicht notwendig. Hinzu kommt, dass Arbeitgebermerkmale wie Branche und Unternehmensgröße als Risikomerkmale in die Prämienkalkulation aufgenommen werden können. Dann müsste allerdings der Versicherungsnehmer zum Teil für das arbeitgeberspezifische Entlassungsverhalten aufkommen.

Auch Gewerkschaften können die Kosten ihrer Tarifpolitik über eine Arbeitslosenversicherung zum Teil externalisieren. Die Durchsetzung hoher Lohnforderungen beispielsweise erhöht ceteris paribus das Arbeitslosigkeitsrisiko (Eekhoff/Milleker 2000: 37 f.). Die Folgen daraus werden über die Arbeitslosenversicherung und nicht von den Tarifparteien finanziert. Bereits 1980 forderte Risch deshalb eine Beteiligung der Gewerkschaften an der Finanzierung der Arbeitslosenversicherung. ${ }^{45}$ Ebenso wie bei dem vorgenannten Moral Hazard stellt sich jedoch die Frage, ob allein die Exis-

45 Dies wird in neuerer Zeit z. B. auch von Berthold (2001: 14) sowie von von Berchem (2005: 280 f.) befürwortet. 
tenz einer Arbeitslosenversicherung zu einem veränderten Verhalten führt; ob die Gewerkschaften also eine aggressivere Lohnpolitik verfolgen nur weil es eine Versicherung gegen die monetären Folgen von Arbeitslosigkeit gibt. Theoretische Gewerkschaftsmodelle, in denen die Gewerkschaft den Nutzen ihrer Mitglieder maximiert, kommen genau zu diesem Ergebnis (Oswald 1982: 586). Hierbei wird allerdings die Finanzierungsseite der Arbeitslosenversicherung vernachlässigt (Berthold/Külp 1987: 64 f.). So würde eine private Versicherung mit risikoäquivalenten Beiträgen, die von den Arbeitnehmern zu entrichten sind, Druck auf eine weniger aggressive Lohnpolitik erzeugen. Gewerkschaftlich organisierte Arbeitnehmer hätten einen Anreiz auf eine Lohnpolitik hinzuwirken, die das durchschnittliche Arbeitslosigkeitsrisiko nicht erhöht (Berthold 1988: 363). Insofern ist ein ausgeprägtes Verhaltensrisiko auf Seiten der Gewerkschaften nicht zu befürchten. ${ }^{46}$

Moral Hazard durch die Versicherungsunternehmen thematisieren Glismann/Schrader (2005: 62). Ausgangspunkt ihrer Argumentation ist ein mangelnder Wettbewerb auf Versicherungsmärkten. Demnach schränken ein staatlich kontrollierter Zugang zum Markt für Arbeitslosenversicherung, Regulierungen bei der Prämienkalkulation sowie Gewinnbeschränkungen den Wettbewerb stark ein. Dies führt letztlich zu überhöhten Preisen für Versicherung. Schadenhöhe und -häufigkeit sind nach Glismann und Schrader jedoch nicht davon betroffen. Dem ist entgegenzuhalten, dass Versicherer auf einem stark regulierten Markt aufgrund der Monopolgewinne weniger Anreize haben, das moralische Risiko zu kontrollieren bzw. zu sanktionieren (Faure 1995: 456). In der Folge steigt das Verhaltensrisiko des Versicherungsnehmers, was letztlich zu höheren und häufigeren Schäden führt. Ausgangspunkt für überhöhte Preise und zu geringe Bekämpfung von Moral Hazard ist allerdings nicht die Existenz einer Versicherung, sondern allzu weit reichende staatliche Eingriffe.

Dies leitet direkt über zu Moral Hazard auf Seiten politischer Entscheidungsträger. Erlässt die Politik Gesetze, die das durchschnittliche Arbeits-

${ }^{46}$ Berthold/Külp (1987: 65) schreiben hierzu: „Finanzieren allein die Arbeitnehmer die Leistungen der Arbeitslosenversicherung wird sich die Lohnpolitik der Gewerkschaften auch bei Existenz einer Arbeitslosenversicherung nicht von der Politik unterscheiden, die sie betreibt, wenn eine materielle Absicherung bei Arbeitslosigkeit über eine Versicherungsinstitution nicht vorhanden ist." 
losigkeitsrisiko ceteris paribus erhöhen oder den Versicherungsmarkt einschränken, so wird dies in der Literatur oftmals als rechtlich-institutionelles Moral Hazard bezeichnet (Sesselmeier et al. 2006: 19 sowie Berthold/ von Berchem 2004: 10). Auch hier kann jedoch in Frage gestellt werden, ob ein Verhaltensrisiko vorliegt. So sind politische Entscheidungen mit negativer Beschäftigungswirkung wohl nicht in erster Linie auf die Existenz einer Arbeitslosenversicherung zurückzuführen (Berthold/von Berchem 2004: 10). Es ist allerdings zu befürchten, dass politische Entscheidungen mit Einfluss auf das durchschnittliche Arbeitslosigkeitsrisiko dahingehend weniger sorgfältig abgewogen werden, wenn privaten Versicherungsunternehmen die Finanzierung obliegt. Dies würde dann sehr wohl ein moralisches Risiko darstellen. Berthold/von Berchem (2004: 10) sehen deshalb im rechtlich-institutionelles Moral Hazard das größte Hindernis für eine private Arbeitslosenversicherung, was auch durch die theoretische Arbeit von Boadway/Marceau (1994) gestützt wird. Dem sind allerdings drei Einwände entgegenzuhalten. Zum einen wird die Politik auch in einem privaten Versicherungssystem in hohem Maße an Arbeitslosenzahlen gemessen (Hartmann 1998: 169). Für politische Entscheidungsträger besteht also in jedem Fall ein großer Anreiz, eine Politik zu betreiben, die das durchschnittliche Arbeitslosigkeitsrisiko nicht erhöht. Zum anderen besitzt das Versicherungsgewerbe bereits heute eine starke Lobby, die durch eine Privatisierung der Arbeitslosenversicherung noch mehr an Einfluss gewinnen würde. Es ist somit davon auszugehen, dass Versicherungsunternehmen ihre Interessen zu vertreten wissen und somit auf eine Politik hinwirken können, die das Risiko der Arbeitslosigkeit zumindest nicht erhöht. Als dritter Einwand kann vorgebracht werden, dass sich rechtlich-institutionelles Moral Hazard wohl kaum durch Prämienerhöhungen verstärkt. Insofern ist diese Form des Verhaltensrisikos in seiner Wirkung gleichzusetzen mit negativen externen Effekten, die lediglich zu einmaligen Prämienanpassungen führen. Diese wären dann von den Versicherungsnehmern zu tragen (Meyer 1997: 21). Die grundsätzliche Versicherbarkeit ist davon nicht betroffen.

Zusammenfassend lässt sich folgendes festhalten: Moral Hazard stellt kein Ausschlusskriterium für eine private Arbeitslosenversicherung dar. Das Verhaltensrisiko beim Versicherungsnehmer, welches allgemein als am gefährlichsten eingestuft wird (Berliner 1982: 100), kann bei einer Ar- 
beitslosenversicherung als geringfügig eingeschätzt werden. ${ }^{47}$ Bisherige Erkenntnisse zur privaten Arbeitslosenzusatzversicherung bestätigen dies (Cebulla 2003: 149 f.). Außerdem haben private Versicherer zahlreiche Instrumente zur Hand, die diese Form des Moral Hazard minimieren. Moralisches Risiko bei Dritten ist am ehesten bei politischen Entscheidungsträgern zu befürchten. Allerdings ist auch hierdurch die grundsätzliche Versicherbarkeit nicht gefährdet.

\subsubsection{Versicherungsnehmer unterschätzen ihr individu- elles Arbeitslosigkeitsrisiko}

Zahlreiche Autoren führen an, dass das individuelle Risiko von Arbeitslosigkeit kollektiv unterschätzt würde (Neubauer/Bäcker 2003: 237; Rürup 1990: 185 f.) und dies vor allem für schlechte Risiken zuträfe (Hartmann 1998: 173; Berthold/von Berchem 2002: 66 und 2004: 7). Bis heute gibt es allerdings keine empirische Evidenz für diese Behauptungen. Zwar können die Arbeiten von Kahneman/Tversky (stellvertretend Tversky/ Kahneman 1982) aufzeigen, dass individuelle Entscheidungen unter Unsicherheit mit Fehlern behaftet sind und individuelle Risiken insofern falsch eingeschätzt werden. Jedoch bedeutet dies keineswegs automatisch eine Unterschätzung (Slovic 1987: 281). Als gesichert gilt dies lediglich für Risken mit geringer Schadenfrequenz und hohem maximalen Schaden wie beispielsweise Katastrophenrisiken (Kunreuther 1976; Tversky/Kahneman 1973, Slovic et al. 1977).

Bisher gibt es keine empirischen Arbeiten, die das subjektive Arbeitslosigkeitsrisiko in Form von echten Wahrscheinlichkeiten erfragen. ${ }^{48}$ Insofern ist ein Vergleich mit dem objektiven Risiko nur sehr vage möglich (Schramm 1992: 59). Hinzu kommt, dass die Arbeitslosenquote als Bestandsgröße keineswegs mit dem Risiko des Arbeitsplatzverlustes, das eine Flussgröße darstellt, gleich zu setzten ist (Franz 2003: 353). Aufgrund

47 Diese Ansicht wird unter anderem von Berthold (1988: 362) sowie Glismann/Schrader (2005: 61) geteilt.

48 Übliche Fragen sind stattdessen: "Do you worry about the security of your present work?" (Yes/No) (Böckerman 2004: 289); "In the future how great a risk do you think there is that you will become unemployed?" (no risk/quite a low risk/quite a high risk/a very high risk) (Ferrera 1993: 23); "Wie wahrscheinlich ist es innerhalb der nächsten zwei Jahre, dass Sie Ihren Arbeitsplatz verlieren?" (ganz sicher/wahrscheinlich/eher unwahrscheinlich/ganz sicher nicht) (Schramm 1992: 60) 
der großen Zahl von Arbeitslosen im Arbeitslosenbestand liegt die Arbeitslosenquote für gewöhnlich weit über der Zuflussquote. Allerdings kann nur letztere als durchschnittliches Arbeitslosigkeitsrisiko im Versicherungskontext interpretiert werden. Insofern ist beispielsweise die Arbeit von Böckerman (2004) nicht gut geeignet, um Aussagen über das Verhältnis von subjektivem zu objektivem Arbeitslosigkeitsrisiko zu treffen. Hier wird für das Jahr 1998 die Angst vor Arbeitplatzverlust in der Kodierung Ja/ Nein der standardisierten Arbeitslosenquote gegenübergestellt (Böckerman 2004: 289). So äußern 36 Prozent der befragten Deutschen eine Angst vor dem Verlust des Arbeitsplatzes. Eine Arbeitslosenquote von 11,2 Prozent kann dazu jedoch in keinerlei Beziehung gesetzt werden. Auch die Untersuchung von Ferrera (1993: 23) liefert keine Anzeichen für eine kollektive Unterschätzung des Arbeitslosigkeitsrisikos. ${ }^{49}$ Es lässt sich lediglich daraus entnehmen, dass etwa 16 Prozent aller befragten Deutschen einer zukünftigen Arbeitslosigkeit ein hohes bis sehr hohes Risiko beimessen. Außerdem liefert die Studie leichte Anhaltspunkte dafür, dass schlechte Risiken ihr subjektives Risiko höher einschätzen als gute Risiken. Ob sie inr individuelles Risiko jedoch „richtig“ bemessen, kann nicht festgestellt werden. Das gleiche gilt für die Auswertungen von Schramm (1992: 60 ff.). Demnach geben 6,8 Prozent der im SOEP 1984-1987 Befragten an, dass sie einen Arbeitsplatzverlust für wahrscheinlich oder sicher halten. Der entsprechende Anteil unter den an- und ungelernten Arbeitern liegt mit 8,1 Prozent deutlich höher.

Der heutige Stand der empirischen Forschung liefert also weder Anhaltspunkte für eine kollektive Unterschätzung des Arbeitslosigkeitsrisikos, noch für eine Unterschätzung speziell bei schlechten Risiken. Wenn überhaupt, dann sind vereinzelte Studien zu nennen, die eine korrekte Einschätzung oder sogar leichte kollektive Überschätzung vermuten lassen (Cebulla 2003: 143 sowie Cebulla et al. 2000: 23 f.). Diese Ergebnisse stehen keineswegs im Widerspruch zu den Erkenntnissen, die Weinstein (1980) als „unrealistic optimism“ bezeichnet. Für negative Ereignisse wie Arbeitsplatzverlust (Weinstein 1980: 810) schätzen Personen demnach ihr individuelles Risiko geringer ein als das von ihnen angenommene Durch-

49 Insofern ist es verwunderlich, dass gerade Hartmann (1998: 172 f.) sowie Berthold/ von Berchem (2002: 66 und 2004: 7) darauf verweisen. 
schnittsrisiko. Da in Weinsteins Untersuchung jedoch sowohl das individuelle als auch das durchschnittliche Risiko der subjektiven Einschätzung des Befragten unterliegt, können daraus keine Schlüsse auf die Beziehung zwischen subjektivem und objektivem Risiko gezogen werden.

\section{Sozialpolitische Argumentation}

Bis zu dieser Stelle wurden die verschiedenen versicherungstechnischen Hemmnisse für die Privatisierung der Arbeitslosenversicherung ausführlich behandelt. Es wurde deutlich, dass diese heute keine Ausschlusskriterien mehr darstellen und somit aus versicherungstechnischer Sicht eine Privatisierung grundsätzlich möglich ist. Die folgenden beiden Abschnitte behandeln die sozialpolitischen Argumente gegen eine Privatisierung. Diese werden damit, entgegen der bisherigen Vorgehensweise in der Literatur, strikt getrennt von der Versicherungstechnik thematisiert. Ausgangspunkt der sozialpolitischen Sichtweise ist die Befürchtung, dass Hochrisikogruppen und damit sozial schlechter gestellte Personen faktisch keinen privaten Versicherungsschutz nachweisen können. Entweder wertschätzen sie diesen nicht ausreichend und fragen somit keine Versicherung nach oder sie können sich keinen Versicherungsschutz leisten. Da es sich hierbei um unterschiedliche Ursachen für das Nichtzustandekommen eines Versicherungskontraktes handelt, werden diese in den beiden folgenden Abschnitten getrennt betrachtet. Letztlich werden dadurch zwei Seiten derselben Medaille angesprochen, weshalb sich einzelne Argumente teilweise ähneln.

\subsection{Die individuelle Wertschätzung einer Arbeitslosen- versicherung ist bei Personen mit hohem Arbeitslo- sigkeitsrisiko zu gering}

Diesem Argument liegt implizit die Beobachtung zugrunde, dass eine risikoäquivalente Prämie zur Arbeitslosenversicherung negativ korreliert ist mit dem verfügbaren Einkommen des Versicherungsnehmers. Außerdem ist zu berücksichtigen, dass die Grundsicherung eine absolute Einkommensuntergrenze darstellt, die gerade für Geringverdiener eine echte Rückfallposition bildet. Somit schätzen Bezieher von geringen Einkommen, welche überwiegend schlechte Risiken darstellen, ihren Nutzen aus der Versicherung ihres Einkommens gegen Arbeitslosigkeit vergleichsweise gering ein. Im Ergebnis werden diese Personen also keine Versicherung nachfragen (Schönbäck 1988: 55). Dieses rationale Desinteresse an der 
Absicherung der monetären Folgen von Arbeitslosigkeit ist insofern problematisch, als dass es negative externe Effekte für die Gemeinschaft der Steuerzahler generiert. So könnte eine erhöhte Inanspruchnahme der steuerfinanzierten Grundsicherung nur über ein größeres Steueraufkommen finanziert werden. Falls dies gesellschaftlich nicht erwünscht ist (Eekhoff/Milleker 2000: 23), kann entweder die Grundsicherung anreizkompatibel umgestaltet werden ${ }^{50}$ oder ein Versicherungszwang eingeführt werden. Ersteres dürfte die Problematik zwar abschwächen, aber nicht systematisch beheben. Eine Zwangsnachfrage nach Versicherung engt die Handlungsfreiheit der Akteure stark ein und ist insofern nur schwerlich kompatibel mit einem privaten Versicherungssystem (Glismann/Schrader 2005: 74). ${ }^{51}$ Trittbrettfahren kann einen derartigen Eingriff jedoch grundsätzlich rechtfertigen (Petersen 1989: 62). Es muss an dieser Stelle offen bleiben, welche der genannten Optionen - (i) Akzeptanz der Grundsicherung als Substitut für eine private Arbeitslosenversicherung, (ii) anreizkompatible Umgestaltung der Grundsicherung, (iii) Einführung eines Versicherungszwangs - realisiert werden sollte. Dies obliegt in erster Linie gesellschaftlichen Wertvorstellungen.

\subsection{Für Personen mit hohem Arbeitslosigkeitsrisiko ist die finanzielle Belastung durch risikoäquivalente Prämien zu hoch}

Ausgangspunkt dieser Argumentation ist ebenfalls der Umstand, dass risikoäquivalente Prämie und Budget negativ korreliert sind. Aufgrund eines relativ schlechten Qualifikationsniveaus erzielen Personen mit hohem Arbeitslosigkeitsrisiko systematisch geringere Einkommen. Nach Abzug eines fixen Existenzminimums verbleibt für diese Personen nur ein geringer bis kein Spielraum zur Finanzierung einer risikoäquivalenten Prämie in der

50 Glismann/Schrader (2005: 52 ff.) geben einen Überblick über unterschiedliche Ausgestaltungsformen der Grundsicherung, welche anreizkompatibel mit einer privaten Arbeitslosenversicherung sind. Einige grundsätzliche Gestaltungsoptionen nennen Berthold/von Berchem (2002: $102 \mathrm{ff}$.): Absenkung des allgemeinen Sicherungsniveaus der Grundsicherung, temporäre Senkung der Transferentzugsrate, temporäre Lohnsubventionierung, Verbesserung der Betreuung und Kontrolle durch Dezentralisierung der Ausgestaltungskompetenzen.

${ }^{51}$ Die Kfz-Haftpflichtversicherung ist an dieser Stelle kein adäquates Beispiel. Dort wird der Versicherungszwang mit dem Schutz des geschädigten Ditten begründet (Farny 2006: 149). 
Arbeitslosenversicherung (Walker et al. 1995: 47). ${ }^{52}$ Problematisch ist dieser Punkt grundsätzlich nur dann, wenn es eine Gesellschaft als wünschenswert erachtet, jede Risikogruppe gegen Arbeitslosigkeit zu versichern. Ansonsten bekämen - wie in der Privatversicherung üblich - eben nur jene Personen Versicherungsschutz, die sich dies auch leisten können. Für den Rest der Gesellschaft wäre dann die Grundsicherung die Sicherungsinstanz.

An dieser Stelle mag es hilfreich sein, sich die eigentlichen Kernaufgaben einer Arbeitslosenversicherung vor Augen zu führen. Zum einen hat sie das Ziel, das Einkommen und damit den Konsum im Lebensverlauf zu verstetigen und hierdurch die gesamtwirtschaftliche Wohlfahrt zu erhöhen (Schäfer 2006: 24). Zweites Ziel ist die Realisierung von Produktivitätsgewinnen durch ein verbessertes Matching am Arbeitsmarkt (Acemoglu/ Shimer 2000). So ermöglichen die Leistungen der Arbeitslosenversicherung eine längere individuelle Suchdauer, weshalb nicht die erstbeste Stelle angenommen werden muss. Es ist jedoch zu bezweifeln, ob eine Arbeitslosenversicherung für Hochrisikoträger tatsächlich diese beiden Aufgaben erfüllen kann. Da Personen mit hohem Arbeitslosigkeitsrisiko tendenziell relativ geringe Markteinkommen erzielen, fällt ihre maximale Versicherungsleistung ebenfalls vergleichsweise gering aus. Oftmals liegt die Grundsicherung für diesen Personenkreis nur knapp unter oder sogar über dem maximalen Leistungsanspruch aus der Arbeitslosenversicherung (Schneider et al. 2002: 34 ff.). ${ }^{53}$ Für schlechte Risiken werden die Verstetigung des Einkommens sowie die Ermöglichung längerer Suchdauern faktisch nicht durch die Arbeitslosenversicherung sondern von der Grundsi-

52 In der Literatur trifft man in diesem Zusammenhang oftmals auf eine Fehlargumentation. So führen Barr (2001: 36) sowie Bruttel (2005: 292) als versicherungstechnisches Hemmnis für eine private Arbeitslosenversicherung an, dass einige potentielle Versicherungsnehmer ein Arbeitslosigkeitsrisiko nahe eins aufweisen. Hierbei kann es sich allerdings nur um eine Verwechslung handeln, denn Schadeneintrittswahrscheinlichkeiten von eins (wie z. B. in der Sterbegeldversicherung) stellen kein Hindernis für die Versicherbarkeit dar, solange andere Schadendimensionen (Zeitpunkt, Höhe) unsicher sind. Die Autoren thematisieren also vielmehr die Belastungswirkung risikoäquivalenter Prämien für einige Typen von Versicherungsnehmern.

53 Insofern kann auch eine Absenkung des Deckungsgrades für diese Risiken die Problematik nicht entschärfen. 
cherung bewältigt. ${ }^{54}$ Insofern ist anzunehmen, dass eine Nicht-Versicherung der schlechten Risiken aufgrund zu hoher risikoäquivalenter Prämien keine echten Nachteile für diese Personengruppe mit sich bringt. ${ }^{55}$

Die grundlegende Problematik einer negativen Korrelation zwischen Belastungswirkung durch risikoäquivalente Prämien und finanzieller Ausstattung des Versicherungsnehmers ist jedoch nicht von der Hand zu weisen. ${ }^{56}$ So müssten Personen mit hohem bis mittlerem Arbeitslosigkeitsrisiko bzw. niedrigem bis mittlerem Einkommen in einem privaten System höhere Prämien bezahlen als gegenwärtig. Dies kann für viele Versicherungsnehmer empfindliche finanzielle Einschnitte bedeuten (Neubauer/Bäcker 2003: 238), wobei zu befürchten ist, dass gerade Familien mit Kindern davon betroffen wären (Bundeszentrale für politische Bildung 2005). Die Korrektur derartiger Verteilungseffekte schreiben Glismann/Schrader (2005: 70) dem Staat zu. Um gesellschaftlich unerwünschte Verteilungsergebnisse zu berichtigen, müssten die Prämien für bestimmte Versichertenkollektive demnach staatlich subventioniert werden. ${ }^{57}$ Damit gehen allerdings Anreizwirkungen einher, die einer privaten Arbeitslosenversicherung entgegenstehen. Da der einzelne Versicherungsnehmer die Prämiensubventionen in sein Kalkül mit einbezieht, hat er ein bei Weitem geringeres Interesse an der Höhe der Prämie. Dies schränkt erstens den Wettbewerb zwischen den Versicherungsunternehmen ein. Zweitens hat es Einfluss auf das schadenrelevante Verhalten des Versicherungsnehmers, was

54 Glismann/Schrader (2005: 193) führen hierzu aus, dass die schlechten Risiken bereits im gegenwärtigen System zu einem Großteil keinen Anspruch auf Arbeitslosengeld erweben und somit auf die Grundsicherung angewiesen sind. So zählt Arbeitslosigkeit heute zu den bedeutendsten Determinanten für den Bezug von Sozialhilfe bzw. Arbeitslosengeld II (Feist 2000: 26 f.).

55 Eisen (1997: 65 f.) macht deutlich, dass bereits in der gegenwärtigen gesetzlichen Arbeitslosenversicherung viele Versicherungsnehmer aufgrund eines zu niedrigen Leistungsniveaus lediglich über die Sozialhilfe bzw. Arbeitslosengeld II abgesichert sind.

56 Zwar behaupten Beenstock (1985) sowie Beenstock/Brasse (1986: 89), dass die Korrelation zwischen Einkommen und risikoäquivalenter Prämie nicht signifikant sei, allerdings werden die Prämien in diesen Arbeiten lediglich nach Alter, Familienstand, Region und Wirtschaftszweig differenziert. Variablen, die den sozioökonomischen Status einer Person erklären können - wie z. B. Bildung, Nationalität oder Stellung im Beruf werden vollkommen vernachlässigt und die geschätzte Korrelation zwischen Einkommen und Prämie damit verwässert.

$57 \mathrm{Ob}$ eine indirekte Subventionierung über die Familienpolitik hinreichend treffgenau wäre, ist insofern fraglich, da die einzelnen Versichertenkollektive jeweils heterogen in Bezug auf die Familienstruktur sind. 
seinerseits zu einer ungünstigeren Schadenverteilung führt. Effizienzgewinne, die durch eine Privatisierung realisiert werden könnten, würden somit teilweise konterkariert. Staatlich subventionierte Prämien sind nicht systemkonform und stellen deshalb keine echte Lösung dar.

\section{Fazit und Ausblick}

Die gegenwärtige Argumentation zur Privatisierung der Arbeitslosenversicherung ist wenig systematisch. Die in dieser Arbeit entwickelte Trennung zwischen objektiv-technischen und normativen Argumenten erleichtert die Beleuchtung der einzelnen Positionen. Somit hat sich gezeigt, dass versicherungstechnische Probleme keine echten Hindernisse für eine private Arbeitslosenversicherung darstellen. Den Versicherungsunternehmen stehen zahlreiche Instrumente der Risikopolitik zur Verfügung, um Probleme wie stochastisch abhängige Einzelrisiken, unzureichende Selektion der Risiken sowie Moral Hazard aufzulösen. Eine Beteiligung der Arbeitgeber und Gewerkschaften an der Finanzierung ist zwar für die Versicherbarkeit unerheblich, über die Sinnhaftigkeit derartiger Gestaltungselemente kann jedoch durchaus diskutiert werden. Eine kollektive Unterschätzung des Arbeitslosigkeitsrisikos, die von einigen Autoren unterstellt wird und letztlich zum Ausbleiben einer Versicherungsnachfrage führen würde, ist nicht zu befürchten. Weitaus stichhaltiger als versicherungstechnische sind sozialpolitische Probleme, die sich durch die Privatisierung der Arbeitslosenversicherung ergeben würden. So drohen im Bereich der Niedrigeinkommensbezieher negative externe Effekte für die Steuerzahler, sofern die Grundsicherung als Substitut für eine Arbeitslosenversicherung fungiert. Diese Problematik könnte allerdings durch die Umgestaltung der Grundsicherung oder die Einführung eines Versicherungszwangs behoben werden. Nicht aus der Welt zu räumen ist dagegen die hohe Belastungswirkung, die durch risikoäquivalente Prämien auf einige Typen von Versicherungsnehmern zukäme. Staatliche Prämiensubventionen können hierbei nicht weiterhelfen, da sie ihrerseits neue Probleme schaffen. Letztlich spricht also nur das Argument "relativer Armut" gegen die Privatisierung der Arbeitslosenversicherung.

Ausgehend von der Portfoliotheorie nach Makowitz (1952) könnte dem Problem der "relativen Armut“ systemimmanent entgegengewirkt werden, indem das individuelle Arbeitslosigkeitsrisiko mit dem individuellen Lang- 
lebigkeitsrisiko gemeinsam versichert wird. ${ }^{58}$ Als einzige unter den großen Lebensrisiken, sind diese beiden Risiken individuell negativ korreliert ${ }^{59}$ und zeitlich voneinander unabhängig.

Weder in der gesetzlichen, noch in der privaten Rentenversicherung werden die Prämien in Deutschland individuell risikoäquivalent berechnet. Somit besteht keine individuelle Äquivalenz zwischen Erwartungswert der Prämien und Erwartungswert der Leistungen (Leinert/Wagner 2001: 76). Berücksichtigt man, dass der sozioökonomische Status einen systematisch positiven Einfluss auf die Lebensdauer hat, so kommt es im gegenwärtigen Rentensystem zu einer Subventionierung der sozial besser Gestellten durch die sozial schlechter Gestellten (Wagner 1985: 194 sowie Reil-Held 2000).

Durch eine kombinierte Versicherung von Arbeitslosigkeit und Langlebigkeit in einem Versicherungsprodukt würde die private Versicherbarkeit des Arbeitslosigkeitsrisikos gestärkt sowie unerwünschten Umverteilungseffekten in der (gesetzlichen und privaten) Rentenversicherung entgegengewirkt. Im Optimalfall wäre die Prämie aus der kombinierten Versicherung für Personen mit hohem Arbeitslosigkeitsrisiko ceteris paribus geringer als im gegenwärtigen System aus gesetzlicher Arbeitslosen- und Rentenversicherung. Das vorgeschlagene System sieht risikoäquivalente Versicherungsprämien für das kombinierte Risiko aus Arbeitslosigkeit und Langlebigkeit vor. ${ }^{60}$ Es steht damit zwischen einer reinen privaten Arbeitslosenversicherung, wie sie von Glismann/Schrader (2005) sowie Beenstock/ Brasse (1986) vorgeschlagen wird, und einem individuellen Kontenmodell,

58 Das gemeinsame Versichern unterschiedlicher Risiken innerhalb eines Versicherungsproduktes, um weitere Diversifikationsmöglichkeiten zu nutzen, wird im Versicherungsgewerbe unter den Begriffen Multiline-Produkte sowie Integrierte Programme thematisiert (vgl. hierzu beispielsweise Eickstädt 2001: 123 ff., Frey/Nießen 2001: 115 ff. sowie Herold/Paetzmann 1999: 49 ff.). Zweifel (1996) weist auf die Vorteile dieses Vorgehens in der Sozialversicherung hin.

59 Diese negative Korrelation besteht über den sozioökonomische Status einer Person (vgl. zum Langlebigkeitsrisiko exemplarisch von Gaudecker/Scholz 2006; Lauterbach et al. 2006, Schneider 2002 sowie zum Arbeitslosigkeitsrisiko Bender et al. 2000; Biewen/Wilke 2005, Steiner 1990).

60 Kohlmann (1996: 381) schlägt bereits die Kombination einer privaten Arbeitslosenversicherung mit einer privaten Rentenversicherung vor. Allerdings geht der Autor dabei nicht von risikoäquivalenten Prämien zur Rentenversicherung aus. Das entsprechende individuelle Diversifikationspotenzial wird in Kohlmanns Vorschlag somit nicht genutzt. 
bei dem positive Kontensalden am Ende des Erwerbslebens verrentet werden. Letzteres wird u. a. von Feldstein/Altmann (1998), Orszag/Snower (2002) sowie Stiglitz/Yun (2002) befürwortet.

\section{Literatur}

Acemoglu, Daron/Shimer, Robert (2000): Productivity Gains from Unemployment Insurance. In: European Economic Review, 44. Jg., H. 7, S. 1195-1224.

Akerlof, George A. (1970): The Markets for Lemons. Quality, Uncertainty and the Market Mechanism. In: Quarterly Journal of Economics, 84. Jg., S. 488-500.

Arrow, Kenneth J. (Hrsg.) (1971): Essays in the Theory of Risk-Bearing. Amsterdam, London.

Arrow, Jairo O. (1996): Estimating the Influence of Health as a Risk Factor on Unemployment. A Survival Analysis of Employment Durations for Workers Surveyed in the German Socio-Economic Panel (1984-1990). In: Social Science \& Medicine, 42. Jg., H. 12, S. 1651-1659.

Arulampalam, Wiji/Stewart, Mark B. (1995): The Determinants of Individual Unemployment Durations in an Era of High Unemployment. In: The Economic J ournal, 105. Jg., S. 321-332.

Barr, Nicholas (1992): Economic Theory and the Welfare State. A Survey and Interpretation. In: J ournal of Economic Literature, 30. Jg., S. 741803.

Barr, Nicholas (Hrsg.) (2001): The Welfare State as Piggy Bank. Information, Risk, Uncertainty, and the Role of the State. Oxford.

Beenstock, Michael (1985): Competitive Unemployment Insurance Pricing. In: Geneva Papers on Risk and Insurance, 10. Jg., H. 34, S. 23-31.

Beenstock, Michael (1986): Competitive Unemployment Insurance in the Market Economy. In: Economic Affairs, 6. Jg., H. 6, S. 10-13.

Beenstock, Michael/Brasse, Valerie (Hrsg.) (1986): Insurance for Unemployment. London: Allen \& Unwin.

Bender, Stefan/Fahrmeir, Ludwig/Lang, Stefan (2000): Determinanten der Arbeitslosigkeitsdauer in Westdeutschland. In: Büchel, Felix/Diewald, Martin/Kraus, Peter/Mertens, Antje/Soga, Heike (Hrsg.) (2000): Zwischen drinnen und draußen. Arbeitsmarktchancen und soziale Ausgrenzung in Deutschland, Opladen, S. 79-94.

Berchem, Sascha von (Hrsg.) (2005): Reform der Arbeitslosenversicherung und Sozialhilfe. Markt, Staat und Föderalismus, Würzburg, Diss. 
Berliner, Baruch (Hrsg.) (1982): Die Grenzen der Versicherbarkeit von Risiken. Zürich.

Berthold, Norbert (1988): Marktversagen, staatliche Intervention und Organisationsformen sozialer Sicherung. In: Rolf, Gabriele/Spahn, Bernd P./Wagner, Gert, S. 339-369.

Berthold, Norbert (Hrsg.) (2001): Der Sozialstaat der Zukunft - mehr Markt, weniger Staat. Würzburg.

Berthold, Norbert/Berchem, Sascha von (Hrsg.) (2002): Kampf gegen Arbeitslosigkeit und Armut. Markt, Staat und Föderalismus. Berlin.

Berthold, Norbert/Berchem, Sascha von (Hrsg.) (2004): Reform der Arbeitslosenversicherung. Markt, Staat oder beides? Würzburg.

Berthold, Norbert/Külp, Bernhard (Hrsg.) (1987): Rückwirkungen ausgewählter Systeme der Sozialen Sicherung auf die Funktionsfähigkeit der Marktwirtschaft. Berlin.

Biewen, Martin/Wilke, Ralf A. (Hrsg.) (2005): Unemployment Duration and the Length of Entitlement Periods for Unemployment Benefits. Do the IAB Employment Subsamle and the German Socio-Economic Panel Yield the Same Results? Mannheim.

Boadway, Robin/Marceau, Nicolas (1994): Time Inconsistency as a Rationale for Public Unemployment Insurance. In: International Tax and Public Finance, 1. Jg., H. 2, S. 107-126.

Böckerman, Petri (2004): Perception of J ob Instability in Europe. In: Social Indicators Research, 67. Jg., H. 3, S. 283-314.

Borch, Karl H./Aase, Knut K./Sandmo, Agnar (Hrsg.) (1990): Economics of Insurance. North-Holland.

Bronars, Stephen G. (1985): Fair Pricing of Unemployment Insurance Premiums. In: The Journal of Business, 58. Jg., H. 1, S. 27-47.

Bruttel, Oliver (2005): Private Versicherungskonten als Reformoption für die öffentliche Arbeitslosenversicherung. In: Sozialer Fortschritt, 54. Jg., H. 12, S. 290-296.

Bundeszentrale für politische Bildung (Hrsg.) (2005): Die soziale Situation in Deutschland. Lebensformen, Familienpolitik - Einkommen von Familien.

[http://www.bdp.de/wissen/QHBRHV,0,0,Einkommen_von_Familien.ht $\mathrm{ml}$, Zugriff am 10.10.2006].

Cebulla, Andreas/Heinelt, Hubert/Walker, Robert (2000): Unemployment and the Insurance Compensation Principle in Britain and Germany. London. 
Cebulla, Andreas (2003): Zur Privatisierung der Arbeitsmarktpolitik in Großbritannien und Deutschland. Raum für private Arbeitslosenversicherung? In: Zeitschrift für Sozialreform, 49. Jg., H. 1, S. 134-152.

Chiu, Henry/Karni, Edi (1998): Endogenous Adverse Selection and Unemployment Insurance. In: J ournal of Political Economy, 106. Jg., H. 4, S. 806-827.

Corak, Miles (1993): Is Unemployment Insurance Addictive? Evidence From the Benefit Durations of Repeat Users. In: Industrial and Labor Relations Review, 47. Jg., H. 1, S. 62-72.

Dionnes, Georges/Lasserre, Pierre (1985): Adverse Selection, Repeated Insurance Contracts and Announcement Strategy. In: Review of Economic Studies, 50. Jg., H. 4, S. 719-723.

Eekhoff, Johann (Hrsg.) (1996): Beschäftigung und soziale Sicherung. Tübingen.

Eekhoff, J ohann/Milleker, David F. (Hrsg.) (2000): Die Aufgaben der Arbeitslosenversicherung neu bestimmen. Bad Homburg.

Eickstädt, Jan (Hrsg.) (2001): Alternative Risiko-Finanzierungsinstrumente und ihr Beitrag zur Lösung aktueller Probleme der Industrieversicherung. München.

Eisen, Roland (1988): Versicherungsprinzip und Umverteilung. Einige theoretische Überlegungen zu den Grenzen des Versicherbaren. In: Rolf, Gabriele/Spahn, Bernd P./Wagner, Gert, S. 117-127.

Eisen, Roland (1997): Reformüberlegungen zur Arbeitslosenversicherung. In: Hauser, Richard (Hrsg.) (1997): Reform des Sozialstaates I. Berlin, S. 45-75.

Eisen, Roland/Zweifel, Peter (Hrsg.) (2003): Versicherungsökonomie. 2. Auflage. Berlin, Heidelberg, New York u.a..

Elias, Peter/Steiner, Victor (Hrsg.) (1998): Labour Markets in Transition. Britain and Germany compared. London.

Farny, Dieter (Hrsg.) (2006): Versicherungsbetriebslehre. 4. Auflage. Karlsruhe.

Faure, Michael G. (1995): The Limits of Insurability from a Law and Economics Perspective. In: Geneva Papers of Risk and Insurance, 20. J g., H. 76, S. 454-462.

Feist, Holger (Hrsg.) (2000): Arbeit statt Sozialhilfe. Zur Reform der Grundsicherung in Deutschland. Tübingen.

Feldstein, Martin (1978): The effect of unemployment insurance on temporary layoff unemployment. In: The American Economic Review, 68. Jg., S. 834-846. 
Feldstein, Martin/Altman, Daniel (Hrsg.) (1998): Unemployment Insurance Savings Accounts. Cambridge.

Ferrera, Maurizio (Hrsg.) (1993): Citizens and Social Protection. Main Results From a Eurobarometer Survey. Brüssel.

Fieldhouse, Ed A. (1996): Putting Unemployment in its Place. Using the Samples of Anonymized Records to Explore the Risk of Unemployment in Great Britain in 1991. In: Regional Studies. Journal of the Regional Studies Association, 30. Jg., H. 2, S. 119-133.

Franz, Wolfgang (1999): Arbeitsmarktökonomik. 4. überarbeitete Auflage. Berlin, Heidelberg, New York u.a..

Frey, Herbert C./Nießen, Gero (2001): Monte Carlo Simulation. Quantitative Risikoanalyse für die Versicherungsindustrie. München.

Gaudecker, Hans-Martin von/Scholz, Rembrandt (2006): Lifetime Earnings and Life Expectancy. Mannheim.

Genosko, J oachim/Hirte, Georg/Weber, Reinhard (1999): Quersubventionierung in der Arbeitslosenversicherung. In: Wirtschaftsdienst, 79. Jg., H. 1, S. 44-49.

Glismann, Hans/Schrader Klaus (2003): Eine effiziente Arbeitslosenversicherung für Deutschland. In: J ahrbücher für die Ordnung von Wirtschaft und Gesellschaft, 54. Jg., S. 143-174.

Glismann, Hans H./Schrader, Klaus (Hrsg.) (2005): Privatisierung der Arbeitslosenversicherung: Ein Konzept für Deutschland. Berlin, Heidelberg, New York.

Grubel, Herbert G. (Hrsg.) (1995): Wohlfahrtsstaat und Effizienz. Gibt es akzeptable Kompromisse? Kiel.

Haley, Joseph D. (Hrsg.) (1990): Using the Financial Markets to Hedge the Risk of Unemployment in the Offering of a Private Unemployment Insurance Product. Lincoln, Diss.

Hartmann, Philipp (Hrsg.) (1998): Grenzen der Versicherbarkeit. Private Arbeitslosenversicherung. Darmstadt, Diss.

Heckman, James J./Borjas, George J.(1980): Does Unemployment Cause Future Unemployment? Definitions, Questions and Answers from a Continuous Time Model of Heterogeneity and State Dependence. In: Economica, 47. Jg., S. 247-283.

Helten, Elmar/Karten, Walter (1984): Das Risiko und seine Kalkulation Teil 1-3. In: Große, Walter/Müller-Lutz, Heinz L./Schmidt, Reimer (Hrsg.) (1984): Versicherungsenzyklopädie. Band 2. Versicherungsbetriebslehre. Wiesbaden, S. 125-275. 
Herold, Bodo/Paetzmann, Karsten (Hrsg.) (1999): Alternativer RisikoTransfer. Die neuen Wege der Industrieversicherung. 2. Auflage. München.

Holz, Michael/Hauser, Hans-Eduard (Hrsg.) (2000): Analyse eines Vorschlags zur Reform der deutschen Arbeitslosenversicherung. Bonn.

Hopenhayn, Hugo A./Nicolini, Juan P. (1997): Optimal unemployment insurance. In: Journal of Political Economy, 105. Jg., H. 2, S. 412-438.

Huber, Christa (2001): Neue Instrumente für massgeschneiderte Versicherungslösungen. In: Winterthur Vorsorge, H. 1, S. 12-15.

Hujer, Reinhard/Schneider, Hilmar (1996): Institutionelle und strukturelle Determinanten der Arbeitslosigkeit in Westdeutschland. Eine mikroökonomische Analyse mit Paneldaten, In: Gahlen, Bernhard/Hesse, Helmut/ Ramser, Hans Jürgen (Hrsg.) (1996): Arbeitslosigkeit und Möglichkeiten ihrer Überwindung. Tübingen, S. 53-76.

Karten, Walter (1972): Zum Problem der Versicherbarkeit und zur Risikopolitik des Versicherungsunternehmens. Betriebswirtschaftliche Aspekte. In: Zeitschrift für die gesamte Versicherungswissenschaft, 61. Jg., S. 279-299.

Knappe, Eckhard (1995): Umbau des Sozialstaates - Kranken-, Rentenund Arbeitslosenversicherung. In: List Forum, 21. Jg., H. 4, S. 342-371.

Kohlmann, Patrick (1996): Individuelle Absicherung vor Arbeitslosigkeit. In: Zeitschrift des Deutschen Sparkassen- und Giroverbandes, 113. Jg., H. 8, S. 381-383.

Kunreuther, Howard C. (1976): Limited Knowledge and Insurance Protection. In: Journal of Public Policy, 2. Jg., S. 237-252.

Lauterbach, Karl W./Lüngen, Markus/Stollwerk, Björn/Gerber, Andreas/ Klever-Deichert, Gabriele (Hrsg.) (2006): Zum Zusammenhang zwischen Einkommen und Lebenserwartung. Köln.

Leinert, J ohannes/Wagner, Gert (2001): Theorie und Empirie steigender Lebenserwartung, Nachreservierung und "Umverteilung" in der gesetzlichen Rentenversicherung. In: Zeitschrift für die gesamte Versicherungswissenschaft, 90. Jg., S. 75-100.

Licht, Georg/Steiner, Victor (1991): Abgang aus der Arbeitslosigkeit, Individualeffekte und Hysteresis. Eine Panelanalyse für die Bundesrepublik Deutschland. In: Helberger, Christof/Bellmann, Lutz/Blaschke, Dieter (Hrsg.) (1991): Erwerbstätigkeit und Arbeitslosigkeit. Analysen auf der Grundlage des Sozioökonomischen Panels. Nürnberg, S. 182-206.

Liebwein, Peter (Hrsg.) (2000): Klassische und moderne Formen der Rückversicherung. Karlsruhe. 
Liebwein, Peter (Hrsg.) (2004): Rückversicherung. Unternehmensplanspiel Versicherungen.

[ http: // www. riva-online.de/download/versplan-net-rueckversicherungd.pdf, Zugriff am 27.04.2006].

Markowitz, Harry (1952): Portfolio Selection. In: The Journal of Finance, 7. Jg., S. 77-91.

Mavromaras, Kostas, G./Rudolph, Helmut (1995): Recalls - Wiederbeschäftigung im alten Betrieb. In: Mitteilungen aus der Arbeitsmarktund Berufsforschung, 28. Jg., H. 2, S. 171-194.

Meyer, Ulrich (1997): Versicherung als Risikotransformation. In: Basedow, Jürgen/Meyer, Ulrich/Schwintowski, Hans-Peter (Hrsg.) (1997): Erneuerung des Versicherungsvertragsgesetzes, Versichertenschutz in den USA, Rechnungslegung von Versicherungsunternehmen. Beiträge der sechsten Wissenschaftstagung des Bundes der Versicherten. BadenBaden, S. 11-26.

Mooij, Ruud A. de (2004): Towards efficient unemployment insurance in the Netherlands.

[http: //www.cpb.nl/nl/memorandum/100/memo100.pdf, Zugriff am 30.04.2005]

Mugler, Josef (1980): Risikopolitische Strategien im Grenzbereich des Versicherbaren. In: Zeitschrift für die gesamte Versicherungswissenschaft, 69. Jg., S. 71-87.

Narendranathan, Wiji/Stewart, Mark B. (1993): Modelling the Probability of Leaving Unemployment. Competing Risks Models with Flexible Baseline Hazards. In: J ournal of the Royal Statistical Society. Applied Statistics, 42. Jg., H. 1, S. 63-83.

Nell, Martin/Richter, Andreas (2003): Catastrophic Events as Threats to Society: Private and Public Risk Management Strategies. In: Frenkel, Michael/Hommel, Ulrich/Rudolf, Markus (Hrsg.) (2003): Risk Management: Challenge and Opportunity. 2. Auflage. Berlin, Heidelberg, S. 321-340.

Neubauer, Jennifer/Bäcker, Gerhard (2003): Abbau der Arbeitslosigkeit durch Abbau der Arbeitslosenversicherung. In: Sozialer Fortschritt, 52. Jg., H. 9, S. 233-239.

Orszag, J. Michael/Snower, Dennis J. (Hrsg.) (2002): From Unemployment Benefits to Unemployment Accounts. Bonn.

Oswald, Andrew J . (1982): The Microeconomic Theory of the Trade Union. In: The Economic Journal, 92. Jg., S. 576-595.

Palan, Dietmar (1996): Kaum Rendite. In: Wirtschaftswoche, H. 5, S. 9293. 
Petersen, Hans-Georg (Hrsg.) (1989): Sozialökonomik. Stuttgart, Berlin, Köln.

Pfeiffer, Christoph (Hrsg.) (1999): Einführung in die Rückversicherung. Das Standardwerk für Theorie und Praxis. 5. Auflage. Wiesbaden.

Reil-Held, Anette (Hrsg.) (2000): Einkommen und Sterblichkeit in Deutschland: Leben Reiche länger? Mannheim.

Risch, Bodo (1980): Arbeitslosenversicherung, Gewerkschaften und Beschäftigungsgrad. In: Die Weltwirtschaft, H. 2, S. 49-57.

Rolf, Gabriele/Spahn, Bernd P./Wagner, Gert (Hrsg.) (1988): Sozialvertrag und Sicherung. Zur ökonomischen Theorie staatlicher Versicherungs- und Umverteilungssysteme. Frankfurt, New York.

Rothschild, Michael/Stiglitz, Joseph E. (1976): Equilibrium in Competitive Insurance Markets. An Essay on the Economics of Imperfect Competition. In: Quarterly Journal of Economics, 90. Jg., S. 629-649.

Rürup, Bert (1990): Sozialpolitik als Produktivkraft. Zur gesamtwirtschaftlichen Effizienz staatlicher Sozialversicherungen. In: Gahlen, Bernhard/ Hesse, Helmut/Ramser Hans Jürgen (Hrsg.) (1990): Theorie und Politik der Sozialversicherungen. Tübingen, S. 179-194.

Rürup, Bert (2005): Arbeitslosenversicherung: Staatlich, privat oder gemischt? In: Zeitschrift für Arbeitsmarktforschung, 2. Jg., H. 3, S. 373282.

Sachverständigenrat (Hrsg.) (2003): Staatsfinanzen konsolidieren - Steuersystem reformieren. Jahresgutachten 2003/2004. Wiesbaden.

Schaaffhausen, Axel von (1989): Grundlagen der Credibility-Theorie. In: Helten, Elmar (Hrsg.) (1989): Beiträge zur Credibility-Theorie. Karlsruhe, S. 11-60.

Schäfer, Holger (Hrsg.) (2003a): Reform der Arbeitslosenversicherung. Ökonomische Aspekte einer politischen Debatte. Köln.

Schäfer, Holger (2003b): Fehlanreize und Reformoptionen in der Arbeitslosenversicherung. In: Sozialer Fortschritt, 52. Jg., H. 9, S. 231-233.

Schäfer, Holger (Hrsg.) (2006): Privatisierung der Arbeitslosenversicherung? Köln.

Schlittgen, Rainer (Hrsg.) (2003): Einführung in die Statistik. Analyse und Modellierung von Daten. 10. Auflage. München, Wien.

Schneider, Sven (Hrsg.) (2002): Lebensstil und Mortalität. Welche Faktoren bedingen ein langes Leben? Wiesbaden.

Schneider, Hilmar/Lang, Cornelia/Rosenfeld, Martin/Kempe, Wolfram/ Kolb, Jürgen (Hrsg.) (2002): Anreizwirkungen der Sozialhilfe auf das Arbeitsangebot im Niedriglohnbereich. Baden-Baden. 
Schneider, Hilmar/Hagedorn, Marcus/Kaul, Ashok/Mennel, Tim (Hrsg.) (2004): Reform der Arbeitslosenversicherung. Benchmarking Deutschland Aktuell, Gütersloh.

Schönbäck, Winfried (1988): Subjektive Unsicherheit als Gegenstand staatlicher Intervention. In: Rolf, Gabriele/Spahn, Bernd P./Wagner, Gert, S. 45-63.

Schramm, Florian (Hrsg.) (1992): Beschäftigungsunsicherheit. Wie sich die Risiken des Arbeitsmarktes auf die Beschäftigten auswirken - Empirische Analysen in Ost und West. Berlin, Diss.

Sesselmeier, Werner/Somaggio, Gabriele/Yollu, Aysel (Hrsg.) (2006): Mögliche Implikationen der gegenwärtigen Arbeitsmarktreformen für die zukünftige Entwicklung der Arbeitslosenversicherung. Gutachten im Auftrag der Hans-Böckler-Stiftung. Landau.

Shavell, Steven/Weiss, Laurence (1979): The Optimal Payment of Unemployment Insurance Benefits over Time. In: The Journal of Political Economy, 87. Jg., H. 6, S. 1347-1362.

Sinn, Hans-Werner (1986): Risiko als Produktivfaktor. In: J ahrbücher für Nationalökonomie und Statistik, 201. Jg., H. 6, S. 557-571.

Slovic, Paul (1987): Perception of Risk. In: Science, 236. Jg., S. 280-285.

Slovic, Paul/Fischhoff, Baruch/Lichtenstein, Sarah/Corrigan, Bernard/ Combs, Barbara (1977): Preference for Insurance against Probable Small Losses: Insurance Implications. In: The J ournal of Risk and Insurance, 44. Jg., H. 2, S. 237-258.

Steiner, Viktor (Hrsg.) (1990): Determinanten individueller Arbeitslosigkeit. Mikroökonomische Erklärungsansätze und empirische Analysen für Österreich. Wien.

Stiglitz, Joseph E. (1983): Risk, Incentives and Insurance: The Pure Theory of Moral Hazard. In: Geneva Papers of Risk and Insurance, 8. Jg., H. 26, S. 4-33.

Stiglitz, Joseph E./Yun, Jungyoll (Hrsg.) (2002): Integration of Unemployment Insurance with Retirement Insurance. Cambridge.

Swiss Re (Hrsg.) (1999): Alternativer Risikotransfer (ART) für Unternehmen. Modeerscheinung oder Risikomanagement des 21. Jahrhunderts? sigma2.

Swiss Re (Hrsg.) (2002): Einführung in die Rückversicherung. Zürich.

Swiss Re (Hrsg.) (2003): Alternativer Risikotransfer - eine Bestandsaufnahme. sigmal.

Swiss Re (Hrsg.) (2005): Innovationen zur Versicherung unversicherbarer Risiken. sigma4. 
Topel, Robert/Welch, Finis (1980): Unemployment Insurance. Survey and Extensions. In: Economica, 47. Jg., S. 351-379.

Tversky, Amos/Kahneman, Daniel (1973): Availability: A Heurisitc for Judging Frequency and Probability. In: Cognitive Psychology, 5. Jg., S. 207-232.

Tversky, Amos/Kahneman, Daniel (1982): Judgement Under Uncertainty. Heuristics and Biases. In: Kahneman, Daniel/Slovic, Paul/Tversky, Amos (Hrsg.) (1982): Judgement Under Uncertainty. Heuristics and Biases, Cambridge, S. 3-20.

Uhlendorff, Arne (Hrsg.) (2003): Der Einfluss von Persönlichkeitseigenschaften und sozialen Ressourcen auf die Arbeitslosigkeitsdauer. Berlin.

Ulrich, Carsten G. (2006): Die soziale Akzeptanz der Arbeitslosenversicherung. In: WSI-Mitteilungen, 59. Jg., H. 4, S. 200-205.

Wagner, Gert (1985): Interpersonelle Umverteilung in der gesetzlichen Rentenversicherung. In: Wirtschaftsdienst, 65. Jg., H. 4, S. 190-196.

Wagner, Thomas/Jahn, Elke (2004): Neue Arbeitsmarttheorien. 2. Auflage. Stuttgart.

Walker, Robert/Shaw, Andrew/Hull, Lisa (1995): Responding to the Risk of Unemployment. In: Association of British Insurers (Hrsg.) (1995): Risk, Insurance and Welfare. The changing balance between public and private protection. London, S. 37-52.

Weinstein, Neil D. (1980): Unrealistic Optimism About Future Life Events. In: Journal of Personality and Social Psychology, 39. Jg., H. 5, S. 806820.

Zweifel, Peter (1996): Konzepte und Kriterien für eine Arbeitsteilung zwischen staatlicher und privater Versicherung. In: Zeitschrift für die gesamte Versicherungswissenschaft, 85. Jg., S. 245-266. 


\section{Recently published}

\begin{tabular}{|c|c|}
\hline No. & Author(s) \\
\hline $1 / 2004$ & $\begin{array}{l}\text { Bauer, T. K. } \\
\text { Bender, S. } \\
\text { Bonin, H. }\end{array}$ \\
\hline $2 / 2004$ & $\begin{array}{l}\text { Achatz, J. } \\
\text { Gartner, H. } \\
\text { Glück, T. }\end{array}$ \\
\hline$\underline{3 / 2004}$ & $\begin{array}{l}\text { Andrews, M. } \\
\text { Schank, T. } \\
\text { Upward, R. }\end{array}$ \\
\hline$\underline{4 / 2004}$ & $\begin{array}{l}\text { Brixy, U. } \\
\text { Kohaut, S. } \\
\text { Schnabel, C. }\end{array}$ \\
\hline$\underline{5 / 2004}$ & $\begin{array}{l}\text { Kölling, A. } \\
\text { Rässler, S. }\end{array}$ \\
\hline$\underline{6 / 2004}$ & $\begin{array}{l}\text { Stephan, G. } \\
\text { Gerlach, K. }\end{array}$ \\
\hline 7/2004 & $\begin{array}{l}\text { Gartner, H. } \\
\text { Stephan, G. }\end{array}$ \\
\hline$\underline{1 / 2005}$ & $\begin{array}{l}\text { Blien, U. } \\
\text { Suedekum, J }\end{array}$ \\
\hline$\underline{2 / 2005}$ & $\begin{array}{l}\text { Brixy, U. } \\
\text { Kohaut, S. } \\
\text { Schnabel, C. }\end{array}$ \\
\hline$\underline{3 / 2005}$ & $\begin{array}{l}\text { Lechner, M. } \\
\text { Miquel, R. } \\
\text { Wunsch, C. }\end{array}$ \\
\hline$\underline{4 / 200}$ & $\begin{array}{l}\text { Hinz, T. } \\
\text { Gartner, H. }\end{array}$ \\
\hline
\end{tabular}

5/2005 Gartner, H. Rässler, S.

6/2005 Alda, $\mathrm{H}$. Bender, S. Gartner, $\mathrm{H}$.
Title

Date

Dismissal protection and worker flows in small establishments

Bonus oder Bias? : Mechanismen geschlechtsspezifischer

Entlohnung

published in: Kölner Zeitschrift für Soziologie und Sozialpsychologie 57 (2005), S. 466-493 (revised)

Practical estimation methods for linked employer-employee data

Do newly founded firms pay lower wages? First evidence from Germany

Editing and multiply imputing German establishment panel data to estimate stochastic production frontier models published in: Zeitschrift für ArbeitsmarktForschung 37 (2004), S. 306-318

Collective contracts, wages and wage dispersion in a multilevel model

How collective contracts and works councils reduce the gender wage gap

Local economic structure and industry development in Germany, 1993-2001

How fast do newly founded firms mature? : empirical analyses on job quality in start-ups published in: Michael Fritsch, Jürgen Schmude (Ed.): Entrepreneurship in the region, New York et al., 2006, S. 95-112

Long-run effects of public sector sponsored training in West Germany

Lohnunterschiede zwischen Frauen und Männern in Branchen, Berufen und Betrieben published in: Zeitschrift für Soziologie 34 (2005), S. 22-39, as: Geschlechtsspezifische Lohnunterschiede in Branchen, Berufen und Betrieben

Analyzing the changing gender wage gap based on multiply imputed right censored wages

The linked employer-employee dataset of the IAB (LIAB) published in: Schmollers Jahrbuch. Zeitschrift für Wirtschaftsund Sozialwissenschaften 125 (2005), S. 327-336, (shortened) as: The linked employer-employee dataset created from the IAB establishment panel and the process-produced data of the IAB (LIAB)

Labour market dynamics from a regional perspective : the multi-account system

Identifying effect heterogeneity to improve the efficiency of job creation schemes in Germany

Wage distributions by wage-setting regime $\quad 4 / 05$

Individual tenure and collective contracts
$7 / 04$

$7 / 04$

$8 / 04$

$9 / 04$

$10 / 04$

$10 / 04$

$12 / 04$

$1 / 05$ 
Stephan, G.

Blien, U.

Hirschenauer, F.

Formula allocation : the regional allocation of budgetary funds

12/2005 Alda, $\mathrm{H}$.

Allaart, $P$.

Bellmann, L.

$\underline{13 / 2005}$ Caliendo, M.

Hujer, R.

Thomsen, S. L.

$\underline{14 / 2005}$ Lechner, M.

Miquel, R.

Wunsch, C.

15/2005 Jensen, U.

Rässler, S.

$\underline{16 / 2005}$ Schnabel, C.

Zagelmeyer, S.

for measures of active labour market policy in Germany

Churning and institutions : Dutch and German establishments

$5 / 05$

compared with micro-level data

Individual employment effects of job creation schemes in

$5 / 05$

Germany with respect to sectoral heterogeneity

The curse and blessing of training the unemployed in a changing economy : the case of East Germany after unification

$6 / 05$

Where have all the data gone? : stochastic production fron-

tiers with multiply imputed German establishment data

published in: Zeitschrift für ArbeitsmarktForschung, Jg. 39, H.

\section{2, 2006, S. 277-295}

Collective bargaining structure and its determinants : an em-

pirical analysis with British and German establishment data

Kohaut, S.

$17 / 2005$ Koch, S.

Stephan, G.

Walwei, $U$.

published in: European Journal of Industrial Relations, Vol.

12 , No. 2. S. $165-188$

Workfare: Möglichkeiten und Grenzen

published in: Zeitschrift für ArbeitsmarktForschung 38 (2005), S. 419-440

$\underline{18 / 2005}$ Alda, $\mathrm{H}$.

Bellmann, L.

Gartner, $\mathrm{H}$.

19/2005 Eichhorst, W.

Konle-Seidl, R.

Wage structure and labour mobility in the West German pri-

vate sector 1993-2000

The interaction of labor market regulation and labor market

policies in welfare state reform

20/2005 Gerlach, K.

Stephan, G.

Tarifverträge und betriebliche Entlohnungsstrukturen

published in: Auf allen Märkten zu Hause, Marburg 2006

$\underline{21 / 2005}$ Fitzenberger, B.

Speckesser, S.

Employment effects of the provision of specific professional

skills and techniques in Germany

22/2005 Ludsteck, J. Jacobebbinghaus, $P$.

$\underline{1 / 2006}$ Gerlach, K

Levine, D.

Stephan, G.

Struck, O.

2/2006 Ludsteck, J.

Strike activity and centralisation in wage setting

$7 / 05$

$8 / 05$

$8 / 05$

$8 / 05$

$9 / 05$

$11 / 05$

$11 / 05$

$12 / 05$

The acceptability of layoffs and pay cuts : comparing North

America with Germany

$1 / 06$

3/2006 Gaggermeier, C.

Employment effects of centralization in wage setting in a me-

$2 / 06$ dian voter model

2/06

ous preferences

4/2006 Binder, J.

Korrekturverfahren zur Berechnung der Einkommen über der

Schwengler, B.

Beitragsbemessungsgrenze

5/2006 Brixy, U.

Grotz, R.

6/2006 Blien, U.

Sanner, $\mathrm{H}$.

7/2006 Stephan, G.

Rässler, S.

Schewe, T.

Regional patterns and determinants of new firm formation

and survival in western Germany

Structural change and regional employment dynamics

$3 / 06$

$4 / 06$

Wirkungsanalyse in der Bundesagentur für Arbeit : Konzepti-

on, Datenbasis und ausgewählte Befunde

$4 / 06$

$4 / 06$

8/2006 Gash, V.

Are fixed-term jobs bad for your health? : a comparison of

$5 / 06$

Mertens, A.

West-Germany and Spain 


\begin{tabular}{|c|c|c|c|}
\hline $10 / 2006$ & $\begin{array}{l}\text { Jahn, E. J. } \\
\text { Wagner, T. }\end{array}$ & $\begin{array}{l}\text { Base period, qualifying period and the equilibrium rate of } \\
\text { unemployment }\end{array}$ & 6/06 \\
\hline $11 / 2006$ & $\begin{array}{l}\text { Jensen, U. } \\
\text { Gartner, H. } \\
\text { Rässler, S. }\end{array}$ & $\begin{array}{l}\text { Measuring overeducation with earnings frontiers and multiply } \\
\text { imputed censored income data }\end{array}$ & $6 / 06$ \\
\hline $12 / 2006$ & $\begin{array}{l}\text { Meyer, B. } \\
\text { Lutz, C. } \\
\text { Schnur, P. } \\
\text { Zika, G. }\end{array}$ & $\begin{array}{l}\text { National economic policy simulations with global interde- } \\
\text { pendencies : a sensitivity analysis for Germany }\end{array}$ & $7 / 06$ \\
\hline $13 / 2006$ & $\begin{array}{l}\text { Beblo, M. } \\
\text { Bender, S. } \\
\text { Wolf, E. }\end{array}$ & $\begin{array}{l}\text { The wage effects of entering motherhood : a within-firm mat- } \\
\text { ching approach }\end{array}$ & $8 / 06$ \\
\hline $14 / 2006$ & Niebuhr, A. & $\begin{array}{l}\text { Migration and innovation : does cultural diversity matter for } \\
\text { regional } R \& D \text { activity? }\end{array}$ & $8 / 06$ \\
\hline $15 / 2006$ & $\begin{array}{l}\text { Kiesl, H. } \\
\text { Rässler, S. }\end{array}$ & How valid can data fusion be? & $8 / 06$ \\
\hline $16 / 2006$ & $\begin{array}{l}\text { Hujer, R. } \\
\text { Zeiss, C. }\end{array}$ & $\begin{array}{l}\text { The effects of job creation schemes on the unemployment } \\
\text { duration in East Germany }\end{array}$ & $8 / 06$ \\
\hline $17 / 2006$ & $\begin{array}{l}\text { Fitzenberger, B. } \\
\text { Osikominu, A. } \\
\text { Völter, R. }\end{array}$ & $\begin{array}{l}\text { Get training or wait? : long-run employment effects of training } \\
\text { programs for the unemployed in West Germany }\end{array}$ & $9 / 06$ \\
\hline $18 / 2006$ & $\begin{array}{l}\text { Antoni, M. } \\
\text { Jahn, E. J. }\end{array}$ & $\begin{array}{l}\text { Do changes in regulation affect employment duration in tem- } \\
\text { porary work agencies? }\end{array}$ & $9 / 06$ \\
\hline $19 / 2006$ & $\begin{array}{l}\text { Fuchs, J. } \\
\text { Söhnlein, D. }\end{array}$ & $\begin{array}{l}\text { Effekte alternativer Annahmen auf die prognostizierte Er- } \\
\text { werbsbevölkerung }\end{array}$ & $10 / 06$ \\
\hline $20 / 2006$ & $\begin{array}{l}\text { Lechner, M. } \\
\text { Wunsch, C. }\end{array}$ & $\begin{array}{l}\text { Active labour market policy in East Germany : waiting for the } \\
\text { economy to take off }\end{array}$ & $11 / 06$ \\
\hline$\underline{21 / 2006}$ & Kruppe, T. & $\begin{array}{l}\text { Die Förderung beruflicher Weiterbildung : eine mikroökono- } \\
\text { metrische Evaluation der Ergänzung durch das ESF-BA- } \\
\text { Programm }\end{array}$ & $11 / 06$ \\
\hline$\underline{22 / 2006}$ & $\begin{array}{l}\text { Feil, M. } \\
\text { Klinger, S. } \\
\text { Zika, G. }\end{array}$ & $\begin{array}{l}\text { Sozialabgaben und Beschäftigung : Simulationen mit drei } \\
\text { makroökonomischen Modellen }\end{array}$ & $11 / 06$ \\
\hline $23 / 2006$ & $\begin{array}{l}\text { Blien, U. } \\
\text { Phan, t. H. V. }\end{array}$ & $\begin{array}{l}\text { A pilot study on the Vietnamese labour market and its social } \\
\text { and economic context }\end{array}$ & $11 / 06$ \\
\hline
\end{tabular}

Letzte Aktualisierung: 27.11.2006, 52 Einträge 


\section{Impressum}

\section{IABDiscussionPaper}

No. 24 / 2006

\section{Herausgeber}

Institut für Arbeitsmarkt- und Berufsforschung der Bundesagentur für Arbeit

Weddigenstr. 20-22

D-90478 Nürnberg

\section{Redaktion}

Regina Stoll, Jutta Palm-Nowak

Technische Herstellung Jutta Sebald

\section{Rechte}

Nachdruck - auch auszugsweise - nur mit Genehmigung des IAB gestattet

\section{Bezugsmöglichkeit}

Volltext-Download dieses DiscussionPaper

unter:

http://doku.iab.de/discussionpapers/2006/dp2406.pdf

\section{IAB im Internet}

http://www.iab.de

Rückfragen zum Inhalt an

Roman Lutz, Tel. 0911/179-5950, oder E-Mail: roman.lutz@iab.de 